\title{
Review Article \\ Theory of Neutrino-Atom Collisions: The History, Present Status, and BSM Physics
}

\author{
Konstantin A. Kouzakov ${ }^{1}$ and Alexander I. Studenikin ${ }^{2,3}$ \\ ${ }^{1}$ Department of Nuclear Physics and Quantum Theory of Collisions, Faculty of Physics, Lomonosov Moscow State University, \\ Moscow 119991, Russia \\ ${ }^{2}$ Department of Theoretical Physics, Faculty of Physics, Lomonosov Moscow State University, Moscow 119991, Russia \\ ${ }^{3}$ Joint Institute for Nuclear Research, Dubna 141980, Russia \\ Correspondence should be addressed to Konstantin A. Kouzakov; kouzakov@srd.sinp.msu.ru
}

Received 14 March 2014; Accepted 17 June 2014; Published 10 July 2014

Academic Editor: Michael A. Schmidt

Copyright @ 2014 K. A. Kouzakov and A. I. Studenikin. This is an open access article distributed under the Creative Commons Attribution License, which permits unrestricted use, distribution, and reproduction in any medium, provided the original work is properly cited. The publication of this article was funded by SCOAP ${ }^{3}$.

\begin{abstract}
An overview of the current theoretical studies on neutrino-atom scattering processes is presented. The ionization channel of these processes, which is studied in experiments searching for neutrino magnetic moments, is brought into focus. Recent developments in the theory of atomic ionization by impact of reactor antineutrinos are discussed. It is shown that the stepping approximation is well applicable for the data analysis practically down to the ionization threshold.
\end{abstract}

\section{Introduction}

In particle physics, the neutrino plays a remarkable role of a "tiny" particle. The scale of neutrino mass $m_{v}$ is much lower than that of the charged fermions $\left(m_{v_{f}} \ll m_{f}, f=e, \mu, \tau\right)$. Interaction of neutrinos with matter is extremely weak as compared to that in the case of other known elementary fermions. According to the Standard Model (SM), it can be mediated only via exchange of the $W^{ \pm}$and $Z^{0}$ bosons. However, the recent development of our knowledge of neutrino mixing and oscillations, supported by the discovery of flavor conversions of neutrinos from different sources (see [1-4]), substantiates the assumption that neutrinos can possess electromagnetic properties and, hence, take part in electromagnetic interactions (see, e.g., the review articles [5-7]). These properties include, in particular, the electric charge, the charge radius, the anapole moment, and the dipole electric and magnetic moments. Such nontypical neutrino features are of particular interest, because they open a door to "new physics" beyond the SM (BSM). In spite of appreciable efforts in searches for neutrino electromagnetic characteristics, up to now there is no experimental evidence favoring their nonvanishing values.
Among the electromagnetic properties of neutrinos, the most studied and well understood theoretically are neutrino magnetic moments (NMM), along with electric dipole moments. For the most recent and complete review on theoretical and experimental aspects of NMM, as well as for the corresponding references, see [7]. The effective Lagrangian, which describes the coupling of NMM to the electromagnetic field $F^{\alpha \beta}$, can be written in the form

$$
L_{\text {int }}=\frac{1}{2} \bar{\psi}_{i} \sigma_{\alpha \beta}\left(\mu_{i j}+\epsilon_{i j} \gamma_{5}\right) \psi_{j} F^{\alpha \beta}+\text { h.c., }
$$

where the magnetic moments $\mu_{i j}$, in the presence of mixing between different neutrino states, are associated with the neutrino mass eigenstates $v_{i}$. The interplay between the magnetic moment and neutrino mixing effects is important. Note that the electric (transition) moments $\epsilon_{i j}$ do also contribute to the coupling. A Dirac neutrino may have nonzero diagonal electric moments in models where $\mathrm{CP}$ invariance is violated. For a Majorana neutrino the diagonal magnetic and electric moments are zero. Therefore, NMM can be used to distinguish between Dirac and Majorana neutrinos [8-10].

In the Standard Model the magnetic moment of a massless neutrino is zero. In the minimal extension of the SM, 
the explicit evaluation of the one-loop contributions to the Dirac NMM in the leading approximation over small parameters $b_{i}=m_{i}^{2} / M_{W}^{2}\left(m_{i}\right.$ are the neutrino masses, $i=$ $1,2,3)$ that however exactly accounts for the parameters $a_{l}=$ $m_{l}^{2} / M_{W}^{2}(l=e, \mu, \tau)$ yields the following result [11-14]:

$$
\mu_{i j}^{D}=\frac{e G_{F} m_{i}}{8 \sqrt{2} \pi^{2}}\left(1+\frac{m_{j}}{m_{i}}\right) \sum_{l=e, \mu, \tau} f\left(a_{l}\right) U_{l j} U_{l i}^{*},
$$

where $U_{l i}$ is the neutrino mixing matrix, and

$$
f\left(a_{l}\right)=\frac{3}{4}\left[1+\frac{1}{1-a_{l}}-\frac{2 a_{l}}{\left(1-a_{l}\right)^{2}}-\frac{2 a_{l}^{2} \ln a_{l}}{\left(1-a_{l}\right)^{3}}\right] .
$$

A Majorana neutrino can also have nondiagonal (transition) magnetic moments $\mu_{i j}^{M}=2 \mu_{i j}^{D}(i \neq j)$. The obtained value for NMM is proportional to the neutrino mass and is, in general, of the order $\sim 10^{-21}-10^{-19} \mu_{B}$.

Much larger values for NMM can be obtained in different other SM extensions (see $[6,7]$ for the detailed discussion). However, there is a problem [15] for any BSM theory of how to get a large NMM value and simultaneously to avoid an unacceptable large contribution to the neutrino mass. Recently, this problem has been reconsidered for a class of BSM theories and it has been shown in a modelindependent way that in principle it is possible to avoid the above mentioned contradiction in the case of Dirac [16] and Majorana [17] neutrinos. It has been shown that in this kind of theoretical models the NMM can naturally reach values of $\sim 10^{-15}-10^{-14} \mu_{B}$. These values are at least two orders of magnitude smaller than the present laboratory experimental limits (see below). There is also a huge gap of many orders of magnitude between these values and the prediction of the minimal extension of the SM. Therefore, if any direct experimental confirmation of nonzero NMM is obtained in the laboratory experiments, it will open a window to "new physics."

The neutrino magnetic moments are being searched in reactor $[18,19]$, accelerator $[20,21]$, and solar $[22,23]$ experiments on low-energy elastic neutrino-electron scattering (for more details see the review articles $[6,7]$ and references therein). The current best upper limit on the NMM value obtained in such direct laboratory measurements is

$$
\mu_{v} \leq 2.9 \times 10^{-11} \mu_{B}
$$

where $\mu_{B}=e /\left(2 m_{e}\right)$ is a Bohr magneton. This bound, which is due to the GEMMA experiment [19] with a HPGe detector at Kalinin nuclear power station, is by an order of magnitude larger than the tightest constraint obtained in astrophysics [24]:

$$
\mu_{v} \leq 3 \times 10^{-12} \mu_{B}
$$

And it by many orders of magnitude exceeds the value derived in the minimally extended SM that includes right-handed neutrinos $[12,14]$ :

$$
\mu_{v} \leq 3 \times 10^{-19} \mu_{B}\left(\frac{m_{v}}{1 \mathrm{eV}}\right),
$$

where $m_{v}$ is a neutrino mass. At the same time, there are different theoretical BSM scenarios that predict much higher $\mu_{v}$ values. For example, the effective NMM value in a class of extra-dimension models can be as large as about $10^{-10} \mu_{B}[25]$. Future higher precision reactor experiments can therefore be used to provide new constraints on large extra-dimensions.

The paper is organized as follows. Section 2 outlines the current status of searches for NMM and the problem of atomic-ionization effects in reactor experiments. Section 3 is devoted to the theoretical background for neutrino scattering on atomic electrons. In Section 4, we discuss the case of neutrino scattering on one bound electron. Hydrogen-like states and a semiclassical limit are considered. Section 5 focuses on ionization of many-electron atoms by neutrino impact. The case of a helium atomic target and the Thomas-Fermi and $a b$ initio approaches are discussed. Finally, Section 6 summarizes this review.

\section{Searches for Neutrino Magnetic Moments of Reactor Antineutrinos}

The strategy of experiments searching for NMM is as follows. One studies an inclusive cross section for elastic (anti)neutrino-electron scattering which is differential in the energy transfer $T$. In the ultrarelativistic limit $m_{v} \rightarrow 0$, it is given by an incoherent sum of the SM contribution $d \sigma_{\mathrm{SM}} / d T$, which is due to weak interaction that conserves the neutrino helicity, and the helicity-flipping contribution $d \sigma_{(\mu)} / d T$, which is due to $\mu_{\nu}$,

$$
\frac{d \sigma}{d T}=\frac{d \sigma_{\mathrm{SM}}}{d T}+\frac{d \sigma_{(\mu)}}{d T} .
$$

The SM term is well documented and is given by [26]

$$
\begin{aligned}
\frac{d \sigma_{\mathrm{SM}}}{d T}= & \frac{G_{F}^{2} m_{e}}{2 \pi} \\
& \times\left[\left(g_{V}+g_{A}\right)^{2}+\left(g_{V}-g_{A}\right)^{2}\left(1-\frac{T}{E_{v}}\right)^{2}\right. \\
& \left.+\left(g_{A}^{2}-g_{V}^{2}\right) \frac{m_{e} T}{E_{v}^{2}}\right],
\end{aligned}
$$

where $E_{v}$ is the incident antineutrino energy, $g_{A}=1 / 2$ and $g_{V}=\left(4 \sin ^{2} \theta_{W}+1\right) / 2$ for $v_{e}$, and $g_{A}=-1 / 2$ and $g_{V}=$ $\left(4 \sin ^{2} \theta_{W}-1\right) / 2$ for $\nu_{\mu}$ and $\nu_{\tau}$, with $\theta_{W}$ being the Weinberg angle. For antineutrinos one must substitute $g_{A} \rightarrow-g_{A}$.

The possibility for neutrino-electron elastic scattering due to NMM was first considered in [27], and the cross section of this process was calculated in [28] (the related brief historical notes can be found in [29]). Here we would like to recall the paper by Domogatsky and Nadezhin [30], where the cross section of [28] was corrected and the antineutrinoelectron cross section was considered in the context of the earlier experiments with reactor antineutrinos of Cowan and Reines [31] and Cowan et al. [32], which were aimed to reveal the NMM effects. Discussions on the derivation of the cross section and on the optimal conditions for bounding 
the NMM value, as well as a collection of the cross section formulas for elastic scattering of neutrinos (antineutrinos) on electrons, nucleons, and nuclei, can be found in $[29,33]$. The result relevant to the $\mu_{\nu}$ component in (7) reads $[29,30,33]$

$$
\frac{d \sigma_{(\mu)}}{d T}=4 \pi \alpha \mu_{\nu}^{2}\left(\frac{1}{T}-\frac{1}{E_{\nu}}\right),
$$

where $\alpha$ is the fine-structure constant. Thus, the two components of the cross section (7) exhibit qualitatively different dependencies on the recoil-electron kinetic energy $T$. Namely, at low $T$ values the SM cross section is practically constant in $T$, while that due to $\mu_{\nu}$ behaves as $1 / T$. This means that the experimental sensitivity to NMM value critically depends on lowering the energy threshold of the detector employed for measurement of the recoil-electron spectrum.

The current reactor experiments with germanium detectors $[18,19]$ have reached threshold values of $T$ as low as few $\mathrm{keV}$ and are to further improve the sensitivity to lowenergy deposition in the detector [34-36]. At low energies, however, one can expect a modification of the free-electron formulas due to the binding of electrons in the germanium atoms, where, for example, the energy of the $K_{\alpha}$ line, $9.89 \mathrm{keV}$, indicates that at least some of the atomic binding energies are comparable to the already relevant to the experiment values of $T$. Thus a proper treatment of the atomic effects in neutrino scattering is necessary and important for the analysis of the current and, even more, of the future data with a still lower threshold. Furthermore, there is no known means of independently calibrating experimentally the response of atomic systems, such as the germanium, to the scattering due to the interactions relevant for the neutrino experiments. Therefore, one has to rely on a pure theoretical analysis in interpreting the neutrino data. For the first time this problem was addressed in [37], where a 2-3-time enhancement of the electroweak cross section in the case of ionization from a $1 s$ state of a hydrogen-like atom with nuclear charge $Z$ had been numerically determined at neutrino energies $E_{v} \sim \alpha Z m_{e} c^{2}$. Subsequent numerical calculations within the relativistic Hartree-Fock method for ionization from inner shells of various atoms showed much lower enhancement ( 5-10\%) of the electroweak contribution [38-43]. It was found that in the scattering on realistic atoms, such as germanium, the so-called stepping approximation works with a very good accuracy. The stepping approach, introduced in [40] from an interpretation of numerical data, treats the process as scattering on individual independent electrons occupying atomic orbitals and suggests that the cross section follows the free-electron behavior in (8) and (9) down to $T$ equal to the ionization threshold for the orbital and that below that energy the electron on the corresponding orbital is "deactivated" thus producing a sharp "step" in the dependence of the cross section on $T$.

The interest in the role of atomic effects was renewed in several more recent papers. The early claim [44] of a significant enhancement of the NMM contribution in the case of germanium due to the atomic effects has been later disproved $[45,46]$ and it was argued $[47-50]$ that the modification of the free-electron formulas (8) and (9) by the atomicbinding effects is insignificant down to very low values of $T$. This conclusion appeared to be also in contradiction to the results of [51], where it was deduced by means of numerical calculations that the $\mu_{\nu}$ contribution to ionization of the helium atomic target by impact of electron antineutrinos strongly enhances relative to the free-electron approximation. However, from calculations performed in [52] it follows that the stepping approximation is well applicable practically down to the ionization threshold for helium.

\section{General Theoretical Framework}

As indicated in the introduction, the most sensitive and widely used method for the experimental investigation of the neutrino electromagnetic properties is provided by direct laboratory measurements of low-energy elastic scattering of neutrinos and antineutrinos with electrons in reactor, accelerator, and solar experiments. In this section, we deliver a theoretical background for such studies.

3.1. Neutrino-Electron Interactions. Let us consider the elastic-scattering process

$$
v+e^{-} \longrightarrow v+e^{-},
$$

where an incident neutrino with energy $E_{v}$ transfers to a free electron, which is initially at rest in the laboratory frame, the energy-momentum $q$. There are two recoil-electron observables: the kinetic energy $T$, which amounts to the energy transfer, and the outgoing angle $\chi$ measured with respect to the incident neutrino direction. In the ultrarelativistic limit $m_{v}=0$, these kinematical variables are related by

$$
\cos \chi=\frac{E_{v}+m_{e}}{E_{v}} \sqrt{\frac{T}{T+2 m_{e}}} .
$$

The maximal value of the kinetic electron energy is thus realized when $\chi=0^{\circ}$ and is given by

$$
T_{\max }=\frac{2 E_{v}^{2}}{2 E_{v}+m_{e}} .
$$

Within the SM, the scattering process (10) takes place due to exchange of the weak bosons, as shown in Figure 1. The $W$-boson channel corresponds to the charged current interaction and is absent in the cases of the muon and tau neutrinos. If $\left|q^{2}\right| \ll m_{W}^{2}$, where $m_{W}$ is the $W$-boson mass, the scattering amplitude is given by [26]

$$
M_{W}=\frac{G_{F}}{\sqrt{2}} \bar{u}_{\nu_{2}} \gamma_{\alpha}\left(1-\gamma_{5}\right) u_{\nu_{1}} \bar{u}_{e_{2}} \gamma^{\alpha}\left(1-\gamma_{5}\right) u_{e_{1}},
$$

where $u_{v_{1}}\left(u_{e_{1}}\right)$ and $u_{v_{2}}\left(u_{e_{2}}\right)$ are initial and final neutrino (electron) spinors. The $Z^{0}$ boson mediates the neutral current interaction. The corresponding scattering amplitude in the case $\left|q^{2}\right| \ll m_{Z}^{2}$, where $m_{Z}$ is the $Z^{0}$-boson mass, reads [26]

$$
M_{Z}=\frac{G_{F}}{\sqrt{2}} \bar{u}_{\gamma_{2}} \gamma_{\alpha}\left(1-\gamma_{5}\right) u_{v_{1}} \bar{u}_{e_{2}} \gamma^{\alpha}\left(g_{V}-g_{A} \gamma_{5}\right) u_{e_{1}}
$$




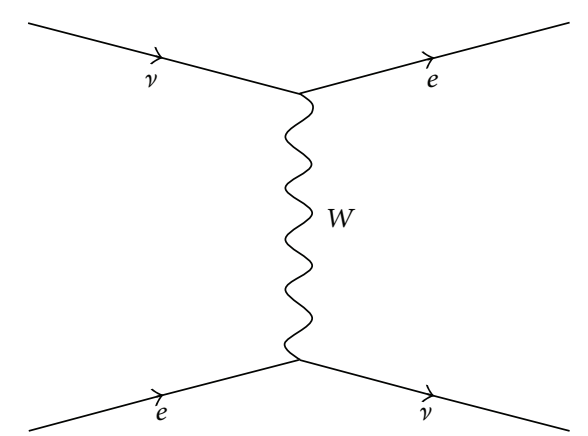

(a)

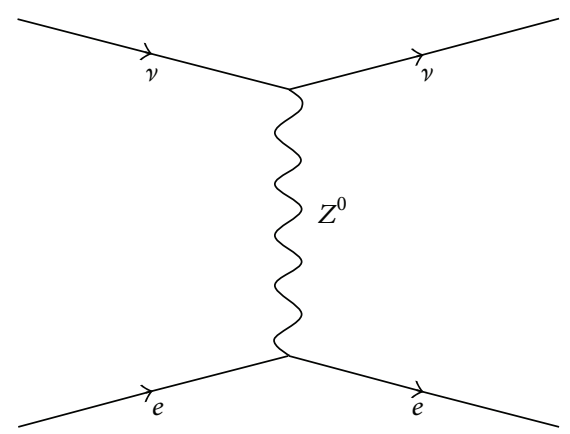

(b)

FIGURE 1: Elastic neutrino-electron scattering due to the weak interaction. Exchange by the $W$ (a) and $Z^{0}$ (b) bosons is shown.

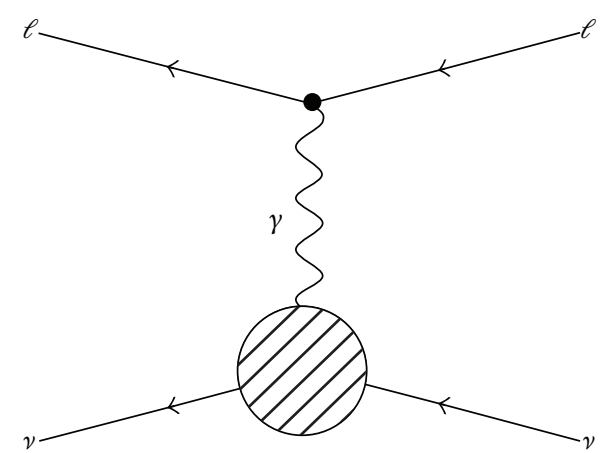

FIGURE 2: Contribution of the neutrino electromagnetic vertex function to neutrino elastic scattering on a charged lepton [5].

Using the matrix elements (13) and (14), one arrives, after averaging over the initial and summing over the final electron spins, at the SM single-differential cross section (8).

Figure 2 shows the electromagnetic channel of the scattering process (10). In general, the matrix element of the neutrino electromagnetic current $J_{\mu}$ can be considered between different neutrino initial $\psi_{i}(p)$ and final $\psi_{j}\left(p^{\prime}\right)$ states of different masses, $p^{2}=m_{i}^{2}$ and $p^{\prime 2}=m_{j}^{2}$,

$$
\left\langle\psi_{j}\left(p^{\prime}\right)\left|J_{\mu}\right| \psi_{i}(p)\right\rangle=\bar{u}_{j}\left(p^{\prime}\right) \Lambda_{\mu}(q) u_{i}(p)
$$

In the most general case consistent with Lorentz and electromagnetic gauge invariance, the vertex function is defined as (see $[5,6]$ and references therein)

$$
\begin{aligned}
\Lambda_{\mu}(q)= & {\left[f_{\mathrm{Q}}\left(q^{2}\right)_{i j}+f_{A}\left(q^{2}\right)_{i j} \gamma_{5}\right]\left(q^{2} \gamma_{\mu}-\gamma_{\mu} q\right) } \\
& +f_{M}\left(q^{2}\right)_{i j} i \sigma_{\mu \nu} q^{\nu}+f_{E}\left(q^{2}\right)_{i j} i \sigma_{\mu \nu} q^{\nu} \gamma_{5},
\end{aligned}
$$

where $f_{Q}\left(q^{2}\right), f_{A}\left(q^{2}\right), f_{M}\left(q^{2}\right)$, and $f_{E}\left(q^{2}\right)$ are, respectively, the charge, anapole, dipole magnetic, and dipole electric neutrino form factors, which are matrices in the space of neutrino mass eigenstates [14].

Consider the diagonal case $i=j$. The hermiticity of the electromagnetic current and the assumption of its invariance under discrete symmetries' transformations put certain constraints on the form factors, which are in general different for the Dirac and Majorana neutrinos. In the case of Dirac neutrinos, the assumption of CP invariance combined with the hermiticity of the electromagnetic current $J_{\mu}$ implies that the electric dipole form factor vanishes, $f_{E}=0$. At zero momentum transfer only $f_{\mathrm{Q}}(0)$ and $f_{M}(0)$, which are called the electric charge and the magnetic moment, respectively, contribute to the Hamiltonian $H_{\text {int }} \sim J_{\mu} A^{\mu}$ that describes the neutrino interaction with the external electromagnetic field $A^{\mu}$. The hermiticity also implies that $f_{Q}, f_{A}$, and $f_{M}$ are real. In contrast, in the case of Majorana neutrinos, regardless of whether CP invariance is violated or not, the charge, dipole magnetic, and electric moments vanish, $f_{\mathrm{Q}}=f_{M}=f_{E}=0$, so that only the anapole moment can be nonvanishing among the electromagnetic moments. Note that it is possible to prove [8-10] that the existence of a nonvanishing magnetic moment for a Majorana neutrino would bring about a clear evidence for CPT violation.

In the off-diagonal case $i \neq j$, the hermiticity by itself does not imply restrictions on the form factors of Dirac neutrinos. It is possible to show [8] that if the assumption of the $\mathrm{CP}$ invariance is added, the form factors $f_{\mathrm{Q}}, f_{M}, f_{E}$, and $f_{A}$ should have the same complex phase. For the Majorana neutrino, if CP invariance holds, there could be either a transition magnetic or a transition electric moment. Finally, as in the diagonal case, the anapole form factor of a Majorana neutrino can be nonzero.

The neutrino dipole magnetic and electric form factors (and the corresponding magnetic and electric dipole moments) are theoretically the most well-understood among the form factors. The value of the magnetic form factor $f_{M}\left(q^{2}\right)$ at $q^{2}=0$ defines the NMM, $\mu_{v}=f_{M}(0)$. In the low-energy limit, the NMM contribution to the effective electromagnetic vertex can be expressed in the following form:

$$
\Lambda_{\alpha}=\frac{\mu_{v}}{2 m_{e}} \sigma_{\alpha \beta} q^{\beta} .
$$

Thus, the corresponding scattering amplitude is

$$
M_{(\mu)}=\frac{4 \pi \mu_{\nu} \sqrt{\alpha}}{2 m_{e} q^{2}} \bar{u}_{v_{2}} \sigma^{\alpha \beta} q_{\beta} u_{v_{1}} \bar{u}_{e_{2}} \gamma_{\alpha} u_{e_{1}} .
$$

This leads to the NMM single-differential cross section given by (9). 
3.2. Neutrino Scattering on Atomic Electrons. Consider the process where a neutrino with energy-momentum $p_{v}=$ $\left(E_{\gamma}, \mathbf{p}_{\gamma}\right)$ scatters on an atom at energy-momentum transfer $q=(T, \mathbf{q})$. In what follows the recoil of the atomic nucleus is neglected because of the typical of current experiments situation $T \gg 2 E_{v}^{2} / M_{N}$, where $M_{N}$ is the nuclear mass. The atomic target is supposed to be unpolarized and in its ground state $|0\rangle$ with the corresponding energy $E_{0}$. It is also supposed that $T \ll m_{e}$ and $\alpha Z \ll 1$, where $Z$ is the nuclear charge and $\alpha$ is the fine-structure constant, so that the initial and final electronic systems can be treated nonrelativistically. The neutrino states are described by the Dirac spinors assuming $m_{v}=0$.

Thus, the magnetic moment interaction of the neutrino field $\psi$ with the atomic electrons is described by the Lagrangian

$$
L_{\mathrm{int}}=\frac{\mu_{\nu}}{2 m_{e}} \bar{\psi}\left(p_{\nu}^{\prime}\right) \sigma_{\alpha \beta} \psi\left(p_{\nu}\right) q^{\alpha} A^{\beta}
$$

where $p_{v}^{\prime}$ is the final neutrino four-momentum. The electromagnetic field $A=\left(A_{0}, \mathbf{A}\right)$ of the atomic electrons is $A_{0}(\mathbf{q})=\sqrt{4 \pi \alpha} \rho(\mathbf{q}) / q^{2}, \mathbf{A}(\mathbf{q})=\sqrt{4 \pi \alpha} \mathbf{j}(\mathbf{q}) / q^{2}$ (hereafter we use the notation $q=|\mathbf{q}|)$, where $\rho(\mathbf{q})$ and $\mathbf{j}(\mathbf{q})$ are the Fourier transforms of the electron number density and current density operators, respectively,

$$
\begin{gathered}
\rho(\mathbf{q})=\sum_{a=1}^{Z} \exp \left(i \mathbf{q} \cdot \mathbf{r}_{a}\right) \\
\mathbf{j}(\mathbf{q})=-\frac{i}{2 m} \sum_{a=1}^{Z}\left[\exp \left(i \mathbf{q} \cdot \mathbf{r}_{a}\right) \frac{\partial}{\partial \mathbf{r}_{a}}+\frac{\partial}{\partial \mathbf{r}_{a}} \exp \left(i \mathbf{q} \cdot \mathbf{r}_{a}\right)\right]
\end{gathered}
$$

and the sums run over the positions $\mathbf{r}_{a}$ of all the $Z$ electrons in the atom. The double-differential cross section can be presented as

$$
\frac{d^{2} \sigma_{(\mu)}}{d T d q^{2}}=\left(\frac{d^{2} \sigma_{(\mu)}}{d T d q^{2}}\right)_{\|}+\left(\frac{d^{2} \sigma_{(\mu)}}{d T d q^{2}}\right)_{\perp},
$$

where

$$
\begin{gathered}
\left(\frac{d^{2} \sigma_{(\mu)}}{d T d q^{2}}\right)_{\|}=4 \pi \alpha \frac{\mu_{v}^{2}}{q^{2}}\left(1-\frac{T^{2}}{q^{2}}\right) S\left(T, q^{2}\right), \\
\left(\frac{d^{2} \sigma_{(\mu)}}{d T d q^{2}}\right)_{\perp}=4 \pi \alpha \frac{\mu_{v}^{2}}{q^{2}}\left(1-\frac{q^{2}}{4 E_{\nu}^{2}}\right) R\left(T, q^{2}\right),
\end{gathered}
$$

where $S\left(T, q^{2}\right)$, also known as the dynamical structure factor [53], and $R\left(T, q^{2}\right)$ are

$$
\begin{aligned}
& S\left(T, q^{2}\right)=\sum_{n} \delta\left(T-E_{n}+E_{0}\right)|\langle n|\rho(\mathbf{q})| 0\rangle|^{2}, \\
& R\left(T, q^{2}\right)=\sum_{n} \delta\left(T-E_{n}+E_{0}\right)\left|\left\langle n\left|j_{\perp}(\mathbf{q})\right| 0\right\rangle\right|^{2},
\end{aligned}
$$

with $j_{\perp}$ being the $\mathbf{j}$ component perpendicular to $\mathbf{q}$ and parallel to the scattering plane, which is formed by the incident and final neutrino momenta. The sums in (25) and (26) run over all the atomic states $|n\rangle$ with energies $E_{n}$ of the electron system, with $|0\rangle$ being the initial state.

The longitudinal term (23) is associated with atomic excitations induced by the force that the neutrino magnetic moment exerts on electrons in the direction parallel to $\mathbf{q}$. The transverse term (24) corresponds to the exchange of a virtual photon which is polarized as a real one, that is, perpendicular to $\mathbf{q}$. It resembles a photoabsorption process when $q \rightarrow T$ and the virtual-photon four-momentum thus approaches a real-photon value. Due to selections rules, the longitudinal and transverse excitations do not interfere (see [54] for detail).

The factors $S\left(T, q^{2}\right)$ and $R\left(T, q^{2}\right)$ are related to, respectively, the density-density (or polarization) and currentcurrent Green's functions

$$
\begin{aligned}
& S\left(T, q^{2}\right)=\frac{1}{\pi} \operatorname{Im} F\left(T, q^{2}\right), \\
& R\left(T, q^{2}\right)=\frac{1}{\pi} \operatorname{Im} L\left(T, q^{2}\right),
\end{aligned}
$$

where

$$
\begin{aligned}
F\left(T, q^{2}\right) & =\sum_{n} \frac{|\langle n|\rho(\mathbf{q})| 0\rangle|^{2}}{T-E_{n}+E_{0}-i \epsilon} \\
& =\left\langle 0\left|\rho(-\mathbf{q}) \frac{1}{T-H+E_{0}-i \epsilon} \rho(\mathbf{q})\right| 0\right\rangle, \\
L\left(T, q^{2}\right) & =\sum_{n} \frac{\left|\left\langle n\left|j_{\perp}(\mathbf{q})\right| 0\right\rangle\right|^{2}}{T-E_{n}+E_{0}-i \epsilon} \\
& =\left\langle 0\left|j_{\perp}(-\mathbf{q}) \frac{1}{T-H+E_{0}-i \epsilon} j_{\perp}(\mathbf{q})\right| 0\right\rangle,
\end{aligned}
$$

$H$ being the Hamiltonian for the system of electrons. From these relations it follows that, due to the parity selection rule, the functions $S\left(T, q^{2}\right)$ and $R\left(T, q^{2}\right)$ are even with respect to $q$.

For small $q$ values, in particular, such that $q \sim T$, only the lowest-order nonzero terms of the expansion of (27) in powers of $q^{2}$ are of relevance (the so-called dipole approximation). In this case, one has $[45,47]$

$$
R\left(T, q^{2}\right)=\frac{T^{2}}{q^{2}} S\left(T, q^{2}\right) .
$$

Taking into account (30), the experimentally measured singedifferential inclusive cross section is, to a good approximation, given by (see, e.g., $[47,49,50])$

$$
\frac{d \sigma_{(\mu)}}{d T}=4 \pi \alpha \mu_{\nu}^{2} \int_{T^{2}}^{\left(2 E_{v}-T\right)^{2}} S\left(T, q^{2}\right) \frac{d q^{2}}{q^{2}} .
$$

The standard electroweak contribution to the cross section can be similarly expressed in terms of the same factor $S\left(T, q^{2}\right)[45,50]$ as

$$
\begin{aligned}
\frac{d \sigma_{\mathrm{SM}}}{d T}= & \frac{G_{F}^{2}}{4 \pi}\left(1+4 \sin ^{2} \theta_{W}+8 \sin ^{4} \theta_{W}\right) \\
& \times \int_{T^{2}}^{\left(2 E_{v}-T\right)^{2}} S\left(T, q^{2}\right) d q^{2}
\end{aligned}
$$


where the factor $S\left(T, q^{2}\right)$ is integrated over $q^{2}$ with a unit weight, rather than $q^{-2}$ as in (31).

The kinematical limits for $q^{2}$ in an actual neutrino scattering are explicitly indicated in (31) and (32). At large $E_{v}$, typical for the reactor neutrinos, the upper limit can in fact be extended to infinity, since in the discussed here nonrelativistic case the range of momenta $q \sim E_{v}$ is indistinguishable from infinity on an atomic scale. The lower limit can be shifted to $q^{2}=0$, since the contribution of the region of $q^{2}<T^{2}$ can be expressed in terms of the photoelectric cross section [45] and is negligibly small (at the level of below one percent in the considered range of $T$ ). For this reason one can discuss the momentum-transfer integrals in (31) and (32) running from $q^{2}=0$ to $q^{2}=\infty$ :

$$
\begin{aligned}
& I_{1}(T)=\int_{0}^{\infty} S\left(T, q^{2}\right) \frac{d q^{2}}{q^{2}}, \\
& I_{2}(T)=\int_{0}^{\infty} S\left(T, q^{2}\right) d q^{2} .
\end{aligned}
$$

For a free electron, which is initially at rest, the densitydensity correlator is the free particle Green's function

$$
F_{(\mathrm{FE})}\left(T, q^{2}\right)=\left(T-\frac{q^{2}}{2 m_{e}}-i \epsilon\right)^{-1},
$$

so that the dynamical structure factor is given by

$$
S_{\text {(FE) }}\left(T, q^{2}\right)=\delta\left(T-\frac{q^{2}}{2 m_{e}}\right),
$$

and the discussed here integrals are in the free-electron limit as follows:

$$
\begin{gathered}
I_{1}^{(\mathrm{FE})}=\int_{0}^{\infty} S_{(\mathrm{FE})}\left(T, q^{2}\right) \frac{d q^{2}}{q^{2}}=\frac{1}{T}, \\
I_{2}^{(\mathrm{FE})}=\int_{0}^{\infty} S_{(\mathrm{FE})}\left(T, q^{2}\right) d q^{2}=2 m_{e} .
\end{gathered}
$$

Clearly, these expressions, when used in the formulas (31) and (32), result in the free-electron cross sections for the case $T \ll$ $E_{\gamma}$

$$
\begin{gathered}
\frac{d \sigma_{(\mu)}}{d T}=\frac{4 \pi \alpha \mu_{v}^{2}}{T}, \\
\frac{d \sigma_{\mathrm{SM}}}{d T}=\frac{G_{F}^{2} m_{e}}{2 \pi}\left(1+4 \sin ^{2} \theta_{W}+8 \sin ^{4} \theta_{W}\right),
\end{gathered}
$$

correspondingly.

\section{Scattering on One Bound Electron}

In this section, we consider neutrino scattering on an electron bound in an atom following consideration of $[47,49,50]$. The binding effects generally deform the density-density Green's function, so that both the integrals (33) are somewhat modified. Namely, the binding effects spread the free-electron $\delta$-peak in the dynamical structure function (35) at $q^{2}=$ $2 m_{e} T$ and also shift it by the scale of characteristic electron momenta in the bound state.
4.1. Ionization from a Hydrogen-Like Orbital. Consider the situation when the initial electron occupies the discrete $n l$ orbital in a Coulomb potential $V(\mathbf{r})=-\alpha Z / r$. The dynamical structure factor for this hydrogen-like system is given by

$$
S_{(n l)}\left(T, q^{2}\right)=\frac{m_{e} k}{(2 \pi)^{3}} \frac{1}{2 l+1} \sum_{m=-l}^{l} \int d \Omega_{k}\left|\left\langle\varphi_{\mathbf{k}}^{-}|\rho(\mathbf{q})| \varphi_{n l m}\right\rangle\right|^{2},
$$

where $\varphi_{n l m}$ is the bound-state wave function, $\varphi_{\mathbf{k}}^{-}$is the outgoing Coulomb wave for the ejected electron with momentum $\mathbf{k}$, and $k=|\mathbf{k}|=\sqrt{2 m_{e} T-p_{n}^{2}}$, with $p_{n}=\alpha Z m_{e} / n$ being the electron momentum in the $n$th Bohr orbit. The closed-form expressions for the bound-free transition matrix elements in (38) can be found, for instance, in [55]. In principle, they allow for performing angular integrations in (38) analytically. This task, however, turns out to be formidable for large values of $n$. Therefore, below we restrict our consideration to the $n=1,2$ states only, which nevertheless is enough for demonstrating the validity of the semiclassical approach described in Section 4.2.

Using results of [56], we can present the function (38) when $n=1,2$ as

$$
\begin{aligned}
S_{(n l)}\left(T, q^{2}\right)= & \frac{2^{8} m_{e} p_{n}^{6}}{3[1-\exp (-2 \pi \eta)]} \\
& \times \frac{q^{2} f_{n l}\left(q^{2}\right)}{\left[\left(q^{2}-k^{2}+p_{n}^{2}\right)^{2}+4 p_{n}^{2} k^{2}\right]^{2 n+1}} \\
& \times \exp \left[-2 \eta \arctan \left(\frac{2 p_{n} k}{q^{2}-k^{2}+p_{n}^{2}}\right)\right],
\end{aligned}
$$

where the branch of the arctangent function that lies between 0 and $\pi$ should be used, $\eta=\alpha Z m_{e} / k$ is the Sommerfeld parameter, and

$$
\begin{aligned}
& f_{1 s}\left(q^{2}\right)=3 q^{2}+k^{2}+p_{1}^{2}, \\
f_{2 s}\left(q^{2}\right)=8[ & 3 q^{10}-\left(32 p_{2}^{2}+11 k^{2}\right) q^{8} \\
& +\left(82 p_{2}^{4}+72 p_{2}^{2} k^{2}+14 k^{2}\right) q^{6} \\
& +\left(20 p_{2}^{6}-62 p_{2}^{4} k^{2}-20 p_{2}^{2} k^{4}-6 k^{6}\right) q^{4} \\
& +\left(p_{2}^{2}+k^{2}\right) \\
& \times\left(\frac{47}{5} p_{2}^{6}-\frac{47}{5} p_{2}^{4} k^{2}-7 p_{2}^{2} k^{4}-k^{6}\right) q^{2} \\
& \left.+\left(4 p_{2}^{2}+k^{2}\right)\left(p_{2}^{2}+k^{2}\right)^{4}\right],
\end{aligned}
$$




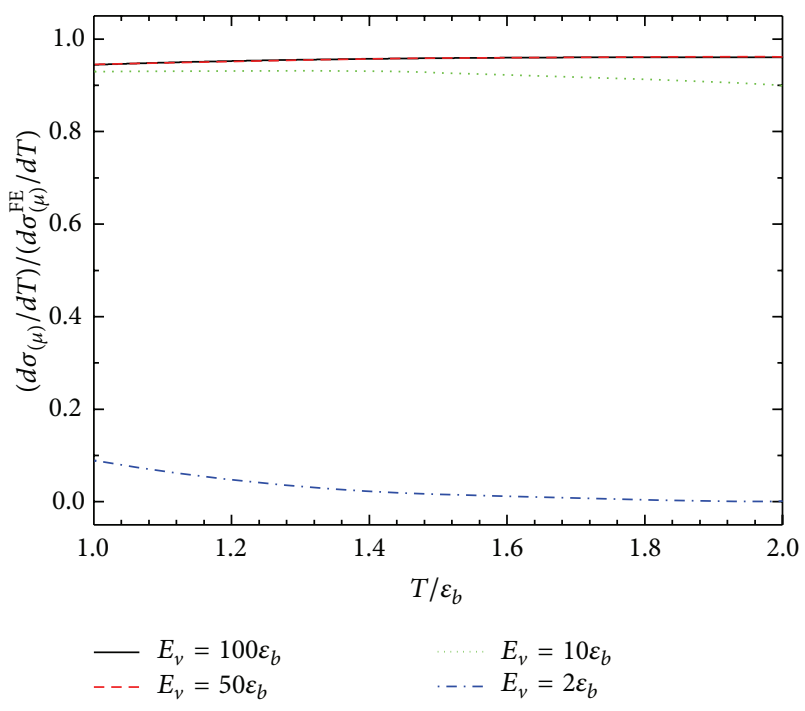

Figure 3: The ratio of single-differential cross sections for magnetic neutrino scattering from the $1 s$ hydrogen-like and free-electron states, respectively, versus $T / \varepsilon_{b}$ at different values of $E_{v}$. The $E_{v}=$ $50 \varepsilon_{b}$ and $E_{v}=100 \varepsilon_{b}$ curves are practically indistinguishable.

$$
\begin{aligned}
f_{2 p}\left(q^{2}\right)=2 p_{2}^{2}[ & 36 q^{8}-48\left(p_{2}^{2}+k^{2}\right) q^{6} \\
& +\left(152 p_{2}^{4}-48 p_{2}^{2} k^{2}-8 k^{4}\right) q^{4} \\
& +\left(p_{2}^{2}+k^{2}\right) \\
& \times\left(\frac{1712}{15} p_{2}^{4}+\frac{1568}{15} p_{2}^{2} k^{2}+16 k^{4}\right) q^{2} \\
& \left.+\left(\frac{44}{3} p_{2}^{2}+4 k^{2}\right)\left(p_{2}^{2}+k^{2}\right)^{3}\right] .
\end{aligned}
$$

Figure 3 shows the magnetic single-differential cross section (31) for ionization from the $1 s$ orbital, which is normalized to the free-electron value (9), as a function of $T / \varepsilon_{b}$, with the electron binding energy given by $\varepsilon_{b}=$ $\alpha^{2} Z^{2} m_{e} / 2$. As can be seen, the numerical results for $E_{v} \gg \varepsilon_{b}$ are close to the free-electron ones in magnitude. This can be qualitatively explained by noticing the following facts. First, in an attractive Coulomb potential there is an infinite set of bound states, with the discrete spectrum smoothly transforming into the continuum at the ionization threshold. Second, the average value of the $1 s$ electron momentum is $p_{e}=p_{1}$ and the average change in the electron momentum after ejection, $\Delta p_{e}$, is such that $\Delta p_{e}^{2}=2 m_{e} T$, which is analogous to the free-electron case.

Thus, taking into account the results in Figure 3, one might expect the atomic-binding effects to play a subsidiary role when $E_{v} \gg \varepsilon_{b}$. The authors of [44], however, came to the contrary conclusion that the single-differential cross section dramatically enhances due to atomic ionization when $T \sim \varepsilon_{b}$. The enhancement mechanism proposed in [44] is based on an analogy with the photoionization process. As mentioned above, when $q \rightarrow T$ the virtual-photon momentum approaches the physical regime $T^{2}-q^{2}=0$.
In this limit, we have for the transverse component of the double-differential cross section (24)

$$
\left(\frac{d^{2} \sigma_{(\mu)}}{d T d q^{2}}\right)_{\perp}=\frac{\mu_{\nu}^{2}}{\pi} \frac{\sigma_{\gamma}(T)}{T}
$$

where $\sigma_{\gamma}(T)$ is the photoionization cross section at the photon energy $T$ [57]. The limiting form (43) was used in [44] in the whole integration interval, when deriving the single-differential cross section. Such procedure is obviously incorrect, for the integrand rapidly falls down as $q^{2}$ ranges from $T^{2}$ up to $\left(2 E_{v}-T\right)^{2}$, especially when $q^{2} \gtrsim r_{a}^{-2}$, where $r_{a}$ is a characteristic atomic size (within the Thomas-Fermi model $\left.r_{a}^{-1}=Z^{1 / 3} \alpha m_{e}[58]\right)$. This fact reflects a strong departure from the real-photon regime. For this reason we can classify the enhancement of the differential cross section determined in [44] as spurious.

Insertion of (39) into the integrals (33) and integration over $q^{2}$, using the change of variable

$$
\frac{2 p_{n} k}{q^{2}-k^{2}+p_{n}^{2}}=\tan x
$$

and the standard integrals involving the products of the exponential function and the powers of sine and cosine functions, yield [50]

$$
\begin{aligned}
& I_{1}^{(1 s)}(T)=\frac{I_{2}^{(1 s)}(T)}{2 m_{e} T} \\
& =\frac{T^{-1}}{1-\exp \left(-2 \pi / \sqrt{y_{1}-1}\right)} \\
& \times\left\{1-\exp \left(-\frac{\pi}{\sqrt{y_{1}-1}}\right)\right. \\
& \times \exp \left[\frac{-2}{\sqrt{y_{1}-1}} \arctan \left(\frac{y_{1}-2}{2 \sqrt{y_{1}-1}}\right)\right] \\
& \left.\times\left(1-\frac{4}{y_{1}}+\frac{16}{3 y_{1}^{2}}\right)\right\} \text {, } \\
& I_{1}^{(2 s)}(T)=\frac{T^{-1}}{1-\exp \left(-4 \pi / \sqrt{y_{2}-1}\right)} \\
& \times\left\{1-\exp \left(-\frac{2 \pi}{\sqrt{y_{2}-1}}\right)\right. \\
& \times \exp \left[\frac{-4}{\sqrt{y_{2}-1}} \arctan \left(\frac{y_{2}-2}{2 \sqrt{y_{2}-1}}\right)\right] \\
& \left.\times\left(1-\frac{8}{y_{2}}+\frac{80}{3 y_{2}^{2}}-\frac{448}{15 y_{2}^{3}}+\frac{1792}{15 y_{2}^{4}}\right)\right\} \text {, }
\end{aligned}
$$




$$
\begin{aligned}
& I_{2}^{(2 s)}(T)=\frac{2 m_{e}}{1-\exp \left(-4 \pi / \sqrt{y_{2}-1}\right)} \\
& \times\left\{1-\exp \left(-\frac{2 \pi}{\sqrt{y_{2}-1}}\right)\right. \\
& \times \exp \left[\frac{-4}{\sqrt{y_{2}-1}} \arctan \left(\frac{y_{2}-2}{2 \sqrt{y_{2}-1}}\right)\right] \\
& \left.\times\left(1-\frac{8}{y_{2}}+\frac{80}{3 y_{2}^{2}}-\frac{448}{15 y_{2}^{3}}+\frac{1024}{15 y_{2}^{4}}\right)\right\} \text {, } \\
& I_{1}^{(2 p)}(T)=\frac{T^{-1}}{1-\exp \left(-4 \pi / \sqrt{y_{2}-1}\right)} \\
& \times\left\{1-\exp \left(-\frac{2 \pi}{\sqrt{y_{2}-1}}\right)\right. \\
& \times \exp \left[\frac{-4}{\sqrt{y_{2}-1}} \arctan \left(\frac{y_{2}-2}{2 \sqrt{y_{2}-1}}\right)\right] \\
& \left.\times\left(1-\frac{8}{y_{2}}+\frac{80}{3 y_{2}^{2}}-\frac{704}{15 y_{2}^{3}}+\frac{3328}{45 y_{2}^{4}}\right)\right\} \text {, } \\
& I_{2}^{(2 p)}(T)=\frac{2 m_{e}}{1-\exp \left(-4 \pi / \sqrt{y_{2}-1}\right)} \\
& \times\left\{1-\exp \left(-\frac{2 \pi}{\sqrt{y_{2}-1}}\right)\right. \\
& \times \exp \left[\frac{-4}{\sqrt{y_{2}-1}} \arctan \left(\frac{y_{2}-2}{2 \sqrt{y_{2}-1}}\right)\right] \\
& \left.\times\left(1-\frac{8}{y_{2}}+\frac{80}{3 y_{2}^{2}}-\frac{704}{15 y_{2}^{3}}+\frac{512}{15 y_{2}^{4}}\right)\right\},
\end{aligned}
$$

where $y_{n}=2 m_{e} T / p_{n}^{2} \equiv T /\left|E_{n}\right|$. The largest deviations of these integrals from the free-electron analogs (36) occur at the ionization threshold $T=\left|E_{n}\right|$. The corresponding relative values in this specific case are [50]

$$
\begin{gathered}
\frac{I_{1}^{(1 s)}}{I_{1}^{(\mathrm{FE})}}=\frac{I_{2}^{(1 s)}}{I_{2}^{(\mathrm{FE})}}=1-\frac{7}{3} e^{-4}=0.9572635093, \\
\frac{I_{1}^{(2 s)}}{I_{1}^{(\mathrm{FE})}}=1-\frac{1639}{15} e^{-8}=0.9633451168, \\
\frac{I_{2}^{(2 s)}}{I_{2}^{(\mathrm{FE})}}=1-\frac{871}{15} e^{-8}=0.9805208034, \\
\frac{I_{1}^{(2 p)}}{I_{1}^{(\mathrm{FE})}}=1-\frac{2101}{45} e^{-8}=0.9843376226, \\
\frac{I_{2}^{(2 p)}}{I_{2}^{(\mathrm{FE})}}=1-\frac{103}{15} e^{-8}=0.9976964900 .
\end{gathered}
$$

The above results indicate a clear tendency: the larger the $n$ and $l$ are, the closer the $I_{1}^{(n l)}$ and $I_{2}^{(n l)}$ are to the free-electron values. The departure from the free-electron behavior does not exceed several percent at most. These observations provide a solid base for the semiclassical approach described below.
4.2. Semiclassical Approach. In the one-electron approximation, the Hamiltonian has the form $H=p^{2} / 2 m_{e}+V(r)$, and the density-density Green's function from (28) can be written as

$$
\begin{aligned}
F & \left(T, q^{2}\right) \\
& =\left\langle 0\left|e^{-i \mathbf{q} \cdot \mathbf{r}}\left[T-H(\mathbf{p}, \mathbf{r})+E_{0}\right]^{-1} e^{i \mathbf{q} \cdot \mathbf{r}}\right| 0\right\rangle \\
& =\left\langle 0\left|\left[T-H(\mathbf{p}+\mathbf{q}, \mathbf{r})+E_{0}\right]^{-1}\right| 0\right\rangle \\
& =\left\langle 0\left|\left[T-\frac{q^{2}}{2 m_{e}}-\frac{\mathbf{p} \cdot \mathbf{q}}{m_{e}}-H(\mathbf{p}, \mathbf{r})+E_{0}\right]^{-1}\right| 0\right\rangle,
\end{aligned}
$$

where the infinitesimal shift $T \rightarrow T-i \epsilon$ is implied.

Clearly, a nontrivial behavior of the latter expression in (53) is generated by the presence of the operator $(\mathbf{p} \cdot \mathbf{q})$ in the denominator and the fact that it does not commute with the Hamiltonian $H$. Thus an analytical calculation of the Green's function as well as the dynamical structure factor is feasible in only few specific problems. In Section 4.1 such calculation has been presented for ionization from the $1 s$, $2 s$, and $2 p$ hydrogen-like states. In particular, we have seen that the deviations of the discussed integrals (33) from their free values are very small: the largest deviation is exactly at the ionization threshold, where, for instance, each of the $1 s$ integrals is equal to the free-electron value multiplied by the factor $\left(1-7 e^{-4} / 3\right) \approx 0.957$ (see (50)). It can be also noted from (45) that both integrals are modified in exactly the same proportion, so that their ratio is not affected at any $T: I_{2}(T) / I_{1}(T)=2 m_{e} T$. We find, however, that this exact proportionality is specific for the ionization from the ground state in the Coulomb potential.

The problem of calculating the integrals (33), however, can be solved in the semiclassical limit, where one can neglect the noncommutativity of the momentum $\mathbf{p}$ with the Hamiltonian and rather treat this operator as a number vector. Taking also into account that $\left(H-E_{0}\right)|0\rangle=0$, one can then readily average the latter expression in (53) over the directions of $\mathbf{q}$ and find the formula for the dynamical structure factor:

$$
\begin{aligned}
& S\left(T, q^{2}\right) \\
& \quad=\frac{m_{e}}{2 p q}\left[\theta\left(T-\frac{q^{2}}{2 m_{e}}+\frac{p q}{m_{e}}\right)-\theta\left(T-\frac{q^{2}}{2 m_{e}}-\frac{p q}{m_{e}}\right)\right],
\end{aligned}
$$

where $\theta$ is the standard Heaviside step function. The expression in (53) is nonzero only in the range of $q$ satisfying the condition $-p q / m_{e}<T-q^{2} / 2 m_{e}<p q / m_{e}$, that is, between the (positive) roots of the binomials in the arguments of the step functions: $q_{\min }^{2}=\sqrt{2 m_{e} T+p^{2}}-p$ and $q_{\max }^{2}=\sqrt{2 m_{e} T+p^{2}}+p$. One can notice that the previously mentioned "spread and shift" of the peak in the dynamical structure function in this limit corresponds to a flat pedestal between $q_{\min }^{2}$ and $q_{\max }^{2}$. The calculation of the integrals (33) 
with the expression (53) is straightforward and yields the freeelectron expressions (36) for the discussed here integrals in the semiclassical (WKB) limit:

$$
I_{1}^{(\mathrm{WKB})}=\frac{1}{T}, \quad I_{2}^{(\mathrm{WKB})}=2 m_{e} .
$$

The appearance of the free-electron expressions here is not surprising, since (53) can be also viewed as the one for scattering on an electron boosted to the momentum $p$. The difference from the pure free-electron case however is in the range of the energy transfer $T$. Namely, the expressions (54) are applicable in this case only above the ionization threshold, that is, at $T \geq\left|E_{0}\right|$. Below the threshold the electron becomes "inactive."

We believe that the latter conclusion explains the socalled stepping behavior observed empirically [40] in the results of numerical calculations. Namely, the calculated cross section $d \sigma / d T$ for ionization of an electron from an atomic orbital follows the free-electron dependence on $T$ all the way down to the threshold for the corresponding orbital with a very small, at most a few percent, deviation. This observation led the authors of [40] to suggest the stepping approximation for the ratio of the atomic cross section (per target electron) to the free-electron one:

$$
f(T) \equiv \frac{d \sigma / d T}{(d \sigma / d T)_{\mathrm{FE}}}=\frac{1}{Z} \sum_{i} n_{i} \theta\left(T-\left|E_{i}\right|\right),
$$

where the sum runs over the atomic orbitals with the binding energies $E_{i}$ and the filling numbers $n_{i}$. Clearly, the factor $f(T)$ simply counts the fraction of "active" atomic electrons at the energy $T$, that is, those for which the ionization is kinematically possible. For this reason we refer to $f(T)$ as an atomic factor. We conclude here that the stepping approximation is indeed justified with a high accuracy in the approximation of the scattering on independent electrons, that is, if one neglects the two-electron correlations induced by the interference of terms in the operator $\rho(\mathbf{q})$ in $(20)$ corresponding to different electrons. The effects of such interference will be discussed in the next section.

\section{Scattering on Many-Electron Atoms}

In considering the neutrino scattering on actual manyelectron atoms one needs to evaluate the dependence of the number of active electrons on $T$ and generally also evaluate the effect of the two-electron correlations. The latter can be studied, for example, in the case of a helium atom, where the electron-electron correlations are known to play a very significant role.

5.1. Helium. Recently, the authors of [51] deduced by means of numerical calculations that the $\mu_{v}$ contribution to ionization of the $\mathrm{He}$ atomic target by impact of electron antineutrinos from reactor and tritium sources strongly departures from the stepping approximation, exhibiting large enhancement relative to the free-electron case. According to [51], the effect is maximal when the $T$ value approaches the ionization threshold in helium, $T_{I}=24.5874 \mathrm{eV}$, where the relative enhancement is as large as almost eight orders of magnitude. It was thus suggested that this finding might have an impact on searches for $\mu_{\nu}$, provided that its value falls within the range $10^{-13}-10^{-12} \mu_{B}$. In this section, following consideration of [52], we show that (i) the result of [51] is erroneous and (ii) the stepping approximation for helium is well applicable, except the energy region $T \sim T_{I}$ where the differential cross section substantially decreases relative to the free-electron case.

We consider the process where an electron antineutrino with energy $E_{v}$ scatters on a He atom at energy and spatialmomentum transfers $T$ and $\mathbf{q}$, respectively. In what follows we focus on the ionization channel of this process in the kinematical regime $T \ll E_{\gamma}$, which mimics a typical situation with reactor $\left(E_{v} \sim 1 \mathrm{MeV}\right)$ and tritium $\left(E_{v} \sim 10 \mathrm{keV}\right)$ antineutrinos when the case $T \rightarrow T_{I}$ is concerned. The He target is assumed to be in its ground state $\left|\Phi_{i}\right\rangle$ with the corresponding energy $E_{i}$. Since for helium one has $\alpha Z \ll 1$, where $Z=2$ is the nuclear charge, the state $\left|\Phi_{i}\right\rangle$ can be treated nonrelativistically. As we are interested in the energy region $T \sim T_{I}$, the final He state $\left|\Phi_{f}\right\rangle$ (with one electron in continuum) can also be treated in the nonrelativistic approximation.

Under the above assumptions, the dynamical structure factor (25) is given by

$$
\begin{aligned}
S\left(T, q^{2}\right)= & \sum_{f}\left|\left\langle\Phi_{f}\left(\mathbf{r}_{1}, \mathbf{r}_{2}\right)\left|e^{i \mathbf{q} \cdot \mathbf{r}_{1}}+e^{i \mathbf{q} \cdot \mathbf{r}_{2}}\right| \Phi_{i}\left(\mathbf{r}_{1}, \mathbf{r}_{2}\right)\right\rangle\right|^{2} \\
& \times \delta\left(T-E_{f}+E_{i}\right) .
\end{aligned}
$$

Here the $f$ sum runs over all final He states having one electron ejected in continuum, with $E_{f}$ being their energies.

For evaluation of the dynamical structure factor (56) we employ the same models of the initial and final He states as in [51]. The initial state is given by a product of two $1 s$ hydrogenlike wave functions with an effective charge $Z_{i}$,

$$
\begin{gathered}
\Phi_{i}\left(\mathbf{r}_{1}, \mathbf{r}_{2}\right)=\varphi_{1 s}\left(Z_{i}, \mathbf{r}_{1}\right) \varphi_{1 s}\left(Z_{i}, \mathbf{r}_{2}\right), \\
\varphi_{1 s}\left(Z_{i}, \mathbf{r}\right)=\sqrt{\frac{Z_{i}^{3}}{\pi a_{0}^{3}} e^{-Z_{i} r / a_{0}}},
\end{gathered}
$$

where $a_{0}=1 /\left(\alpha m_{e}\right)$ is the Bohr radius. The final state has the form

$$
\begin{aligned}
\Phi_{f}\left(\mathbf{r}_{1}, \mathbf{r}_{2}\right) \\
\quad=\frac{1}{\sqrt{2}}\left[\varphi_{\mathbf{k}}^{-}\left(Z_{f}, \mathbf{r}_{1}\right) \varphi_{1 s}\left(Z, \mathbf{r}_{2}\right)+\varphi_{\mathbf{k}}^{-}\left(Z_{f}, \mathbf{r}_{2}\right) \varphi_{1 s}\left(Z, \mathbf{r}_{1}\right)\right],
\end{aligned}
$$

where $\varphi_{\mathbf{k}}^{-}\left(Z_{f}, \mathbf{r}\right)$ is an outgoing Coulomb wave for the ejected electron with spatial momentum $\mathbf{k} . Z_{f}$ is the effective charge experienced by the ejected electron in the field of the final $\mathrm{He}^{+}$ion. Contributions to the dynamical structure factor from excited $\mathrm{He}^{+}$states are neglected due to their very small overlap with the $K$-electron state in the $\mathrm{He}$ atom. 
To avoid nonphysical effects connected with nonorthogonality of states (57) and (58), we use the Gram-Schmidt orthogonalization

$$
\left|\Phi_{f}\right\rangle \longrightarrow\left|\Phi_{f}\right\rangle-\left\langle\Phi_{i} \mid \Phi_{f}\right\rangle\left|\Phi_{i}\right\rangle
$$

Substitution of (57) and (58) into (56) thus yields

$$
\begin{aligned}
S\left(T, q^{2}\right)= & \int \frac{d \mathbf{k}}{(2 \pi)^{3}}|F(\mathbf{k}, \mathbf{q})|^{2} \\
& \times \delta\left(T-\frac{k^{2}}{2 m_{e}}+2 \alpha^{2} m_{e}-Z_{i}^{2} \alpha^{2} m_{e}\right),
\end{aligned}
$$

where $k=\sqrt{2 m_{e}\left(T+2 \alpha^{2} m_{e}-Z_{i}^{2} \alpha^{2} m_{e}\right)}$, and

$$
\begin{aligned}
F(\mathbf{k}, \mathbf{q})= & \sqrt{2}\left\langle\varphi_{\mathbf{k}}^{-}\left(Z_{f}, \mathbf{r}_{1}\right) \varphi_{1 s}\left(Z, \mathbf{r}_{2}\right)\right| e^{i \mathbf{q} \cdot \mathbf{r}_{1}}+e^{i \mathbf{q} \cdot \mathbf{r}_{2}} \\
& -2 \rho_{1 s}(\mathbf{q})\left|\varphi_{1 s}\left(Z_{i}, \mathbf{r}_{1}\right) \varphi_{1 s}\left(Z_{i}, \mathbf{r}_{2}\right)\right\rangle
\end{aligned}
$$

is the inelastic form factor, with

$$
\rho_{1 s}(\mathbf{q})=\int \varphi_{1 s}\left(Z_{i}, \mathbf{r}\right) e^{i \mathbf{q} \cdot \mathbf{r}} \varphi_{1 s}\left(Z_{i}, \mathbf{r}\right) d \mathbf{r}
$$

It is straightforward to perform the further calculation of the dynamical structure factor analytically (see, e.g., the textbook [58]). The resulting expression is

$$
\begin{aligned}
S\left(T, q^{2}\right)= & \frac{2^{16} \alpha^{4} m_{e}^{5} Z_{f} Z_{i}^{6}}{\left(1-e^{2 \pi \eta}\right)\left(2+Z_{i}\right)^{6}} \\
& \times\left[A_{1}(k, q)+B(k, q) A_{2}(k, q)+B^{2}(k, q)\right],
\end{aligned}
$$

where $\eta=-\alpha Z_{f} m_{e} / k$ and (introducing $p_{i}=\alpha Z_{i} m_{e}$ )

$$
\begin{gathered}
A_{1}(k, q) \\
=\left(\left(\exp \left(2 \eta \arccos \frac{p_{i}^{2}+q^{2}-k^{2}}{\sqrt{\left(p_{i}^{2}+q^{2}+k^{2}\right)^{2}-4 k^{2} q^{2}}}\right)\right)\right. \\
\left.\times\left(\left[\left(p_{i}^{2}+q^{2}+k^{2}\right)^{2}-4 k^{2} q^{2}\right]^{3}\right)^{-1}\right) \\
\times\left\{\left(p_{i}+\eta k\right)^{2}\left(p_{i}^{2}+q^{2}+k^{2}\right)^{2}\right) \\
+4 k q^{2}\left[\frac{1}{3} k p_{i}^{2}-\frac{2}{3} \eta^{2} k p_{i}^{2}-\eta^{2} k^{3}\right. \\
\left.\left.-\eta p_{i}\left(p_{i}^{2}+q^{2}+k^{2}\right)\right]\right\}
\end{gathered}
$$

$$
\begin{aligned}
A_{2}(k, q) & \left(\left(2 \exp \left(\eta \arccos \frac{p_{i}^{2}+q^{2}-k^{2}}{\sqrt{\left(p_{i}^{2}+q^{2}+k^{2}\right)^{2}-4 k^{2} q^{2}}}\right)\right)\right. \\
= & \left.\left(\left(p_{i}^{2}+q^{2}+k^{2}\right)^{2}-4 k^{2} q^{2}\right)^{-1}\right) \\
\times & {\left[p_{i} \cos \left(\frac{\eta}{2} \ln \frac{(k+q)^{2}+p_{i}^{2}}{(k-q)^{2}+p_{i}^{2}}\right)\right.} \\
& \left.+\frac{p_{i}^{2}-q^{2}+k^{2}}{2 q}\right) \\
& \left.\times \sin \left(\frac{\eta}{2} \ln \frac{(k+q)^{2}+p_{i}^{2}}{(k-q)^{2}+p_{i}^{2}}\right)\right], \\
B(k, q)= & e^{2 \eta \operatorname{arctg}\left(k / p_{i}\right)} \frac{\left(Z_{i}-Z_{f}\right) \alpha m_{e}}{\left(k^{2}+p_{i}^{2}\right)^{2}} \\
& \times\left\{\frac{\left(2+Z_{i}\right)^{4} \alpha^{4} m_{e}^{4}}{\left[\left(2+Z_{i}\right)^{2} \alpha^{2} m_{e}^{2}+q^{2}\right]^{2}}-\frac{32 p_{i}^{4}}{\left(4 p_{i}^{2}+q^{2}\right)^{2}}\right\} .
\end{aligned}
$$

Finally, the usual choice of the effective charges is $Z_{i}=$ $27 / 16 \approx 1.69$ and $Z_{f}=1$ (see, e.g., [59] and references therein). The value $Z_{i}=27 / 16$ follows from the variational procedure that minimizes the ground-state energy $E_{i}$, while the value $Z_{f}=1$ ensures the correct asymptotic behavior of the final state. However, the authors of [51] utilized in their calculations the values $Z_{i}=1.79$ and $Z_{f}=1.1$ derived from fitting the photoionization cross section data on helium with the present model of the He states.

The departures of the differential cross sections (31) and (32) from the free-electron approximation are characterized by the respective atomic factors

$$
f_{\mathrm{SM}}=\frac{d \sigma_{\mathrm{SM}} / d T}{d \sigma_{\mathrm{SM}}^{\mathrm{FE}} / d T}, \quad f_{\mathrm{NMM}}=\frac{d \sigma_{(\mu)} / d T}{d \sigma_{(\mu)}^{\mathrm{FE}} / d T},
$$

where $d \sigma_{\mathrm{SM}}^{\mathrm{FE}} / d T$ and $d \sigma_{(\mu)}^{\mathrm{FE}} / d T$ are the $\mathrm{SM}$ and $\mu_{\nu}$ contributions to the differential cross section for scattering of an electron antineutrino on two free electrons. Let us recall that following [51] one should expect the $f_{\mathrm{NMM}}$ value to be of about $10^{8}$ at $T \rightarrow T_{I}$.

Numerical results for atomic factors (65) are shown in Figure 4 . They correspond to the kinematical regime $T \ll$ $\alpha m_{e} \ll 2 E_{v}$, which is typically realized both for reactor and for tritium antineutrinos when $T<200 \mathrm{eV}$. Note that in such case one can safely set the upper limit of integrals in (31) and (32) to infinity, as the dynamical structure factor $S\left(T, q^{2}\right)$ rapidly falls down when $q \gtrsim \alpha m_{e}$ and practically vanishes in the region $q \gg \alpha m_{e}$. It can be seen from Figure 4 that atomic factors exhibit similar behaviors for both sets of the $Z_{i}$ and $Z_{f}$ parameters discussed in the previous section. 


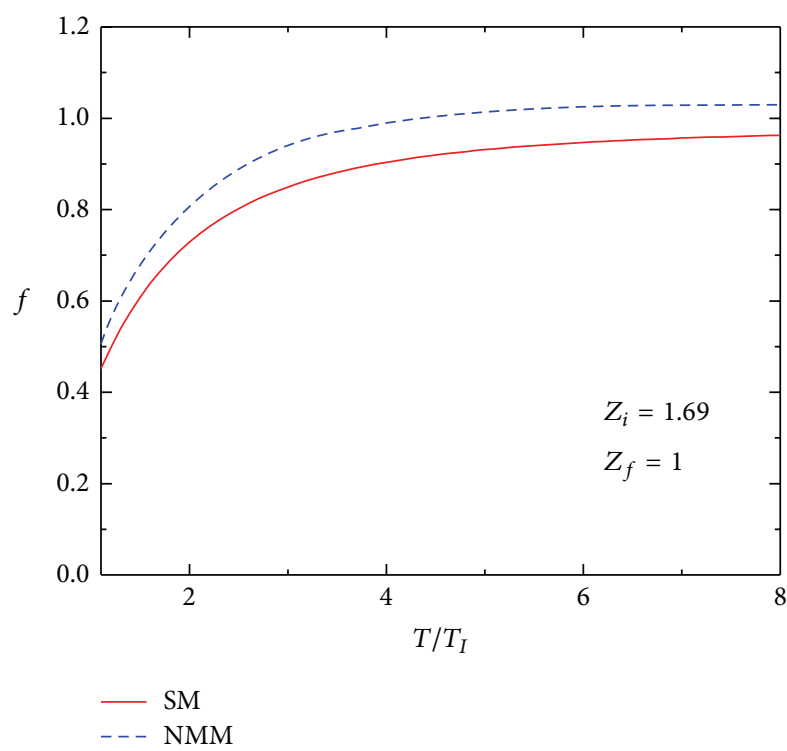

(a)

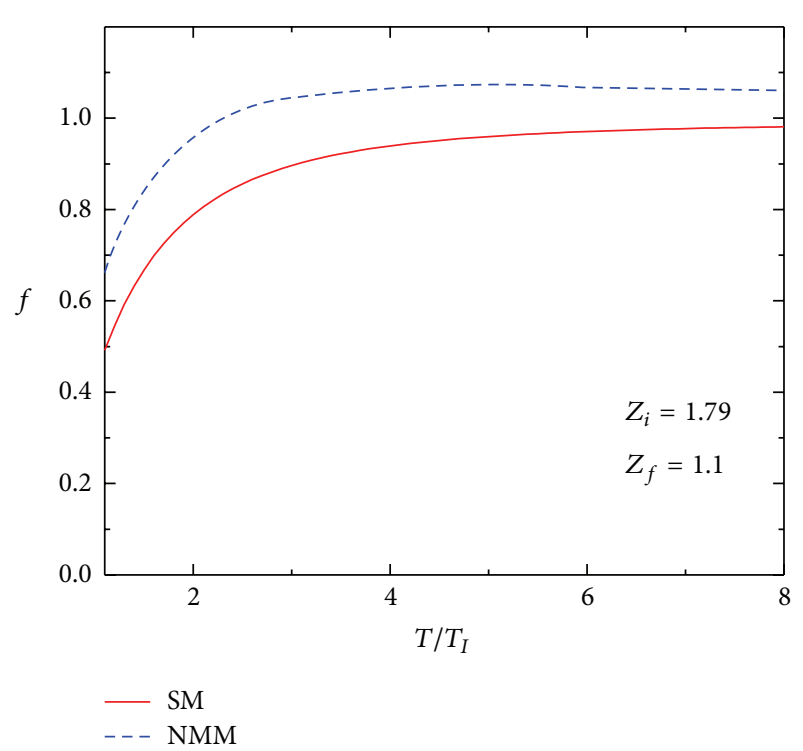

(b)

Figure 4: Atomic factors (65) as functions of the energy transfer [52].

Namely, their values are minimal $(\sim 0.5)$ at the ionization threshold, $T=T_{I}$, and tend to unity with increasing $T$. The latter tendency is readily explained by approaching the freeelectron limit. It can be also seen that a more or less serious deviation $(>10 \%)$ of the present results from the stepping approximation is observed only in the low-energy region $T<$ $100 \mathrm{eV}$. This deviation can be attributed to the effect of the electron-electron correlation in a helium atom. Indeed, if the electrons do not interact with each other, then they occupy two $1 s$ hydrogen-like states (with opposite spins), in which case the departure of the atomic-factor values from unity is, according to the results of Section 4.1, less than 5\%.

Thus, the calculations presented in Figure 4 do not confirm the huge enhancement of the $\mu_{\nu}$ contribution with respect to the free-electron approximation. Moreover, in accord with various calculations for other atomic targets [38$41,43,47,49,50]$, we find that at small energy-transfer values the electron binding in helium leads to the appreciable reduction of the differential cross section relative to the freeelectron case. We attribute the erroneous prediction of [51] to the incorrect dynamical model that draws an analogy between the NMM-induced ionization and photoionization. Indeed, as discussed in Section 3.2, the virtual photon in the NMM-induced ionization process can be treated as real only when $q \rightarrow T$. However, the integration in (31) involves the $q$ values ranging from $T$ up to $2 E_{v}-T$. Since $E_{\gamma} \gg T$, the realphoton picture appears to be applicable only in the vicinity of the lower integration limit. When moving away from that momentum region, one encounters a strong departure from the real-photon approximation which treats the integrand as a constant in the whole integration range, assuming it to be equal to its value at $q=T$; that is,

$$
\frac{1}{q^{2}} S\left(T, q^{2}\right)=\frac{1}{T^{2}} S\left(T, T^{2}\right)
$$

Such an approach is manifestly unjustified, and it gives rise to the spurious enhancement of the $\mu_{\nu}$ contribution to the differential cross section.

5.2. Thomas-Fermi Model. In the Thomas-Fermi model (see, e.g., [58]) the atomic electrons are described as a degenerate free-electron gas in a master potential $\phi(r)$ filling the momentum space up to the zero Fermi energy, namely, up to the momentum $p_{0}(r)$ such that $p_{0}^{2} / 2 m_{e}-e \phi=0$. The electron density $n(r)=p_{0}^{3} /\left(3 \pi^{2}\right)$ then determines the potential $\phi(r)$ from the usual Poisson's equation. In the discussed picture at an energy transfer $T$ the ionization is possible only for the electrons whose energies in the potential are above $-T$, that is, with momenta above $p_{T}(r)$ with $p_{T}^{2} / 2 m_{e}-e \phi=-T$. The electrons with lower energy are inactive. Calculating the density of the inactive electrons as $p_{T}^{3} /\left(3 \pi^{2}\right)$ and subtracting their total number from $Z$, one readily arrives at the formula for the atomic factor, that is, the effective fraction of the active electrons $Z_{\mathrm{eff}} / Z$ as a function of $T$,

$$
\begin{aligned}
f(T) & =\frac{Z_{\text {eff }}(T)}{Z} \\
& =1-\int_{0}^{x_{0}(T)}\left[\frac{\chi(x)}{x}-\frac{T}{T_{0}}\right]^{3 / 2} x^{2} d x,
\end{aligned}
$$

where $\chi(x)$ is the Thomas-Fermi function, well known and tabulated, of the scaling variable $x=2(4 / 3 \pi)^{2 / 3} m_{e} \alpha Z^{1 / 3}$, the energy scale $T_{0}$ is given by

$$
T_{0}=2\left(\frac{4}{3 \pi}\right)^{2 / 3} m_{e} \alpha^{2} Z^{4 / 3} \approx 30.8 Z^{4 / 3} \mathrm{eV},
$$

and, finally, $x_{0}(T)$ is the point where the integrand becomes zero, namely, corresponding to the radius beyond which all 


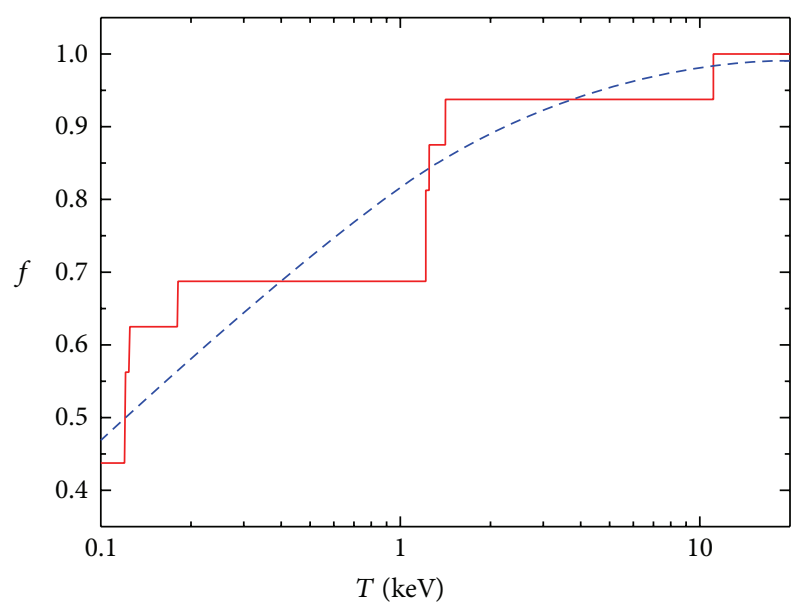

Figure 5: The atomic factor $f$ for germanium in the stepping approximation with the actual energies of the orbitals (solid line) and its interpolation in the Thomas-Fermi model (dashed line) [49].

the electrons are active at the given energy $T$. The energy scale $T_{0}$ in germanium $(Z=32)$ evaluates to $T_{0} \approx 3.1 \mathrm{keV}$. The Thomas-Fermi atomic factor for germanium calculated from the formula (67) is shown by the dashed line in the plot of Figure 5. The discussed statistical model is known to approximate the average bulk properties of the atomic electrons with a relative accuracy $O\left(Z^{-2 / 3}\right)$ and as long as the essential distances $r$ satisfy the condition $Z^{-1} \ll m_{e} \alpha r \ll$ 1 , which condition in terms of the scaling variable $x$ reads as $Z^{-2 / 3} \ll x \ll Z^{1 / 3}$. In terms of the formula (67) for the number of active electrons, the lower bound on the applicability of the model is formally broken at $T \sim Z^{2 / 3} T_{0}$, that is, at the energy scale of the inner atomic shells. However, the effect of the deactivation of the inner electrons is small, of order $Z^{-1}$ in comparison with the total number $Z$ of the electrons. On the other hand, at low $T$, including the most interesting region of $T \sim T_{0}$, the integral in (67) is determined by the range of $x$ of order one, where the model treatment is reasonably justified.

The energies of the inner $K, L$, and $M$ orbitals in the germanium atom are well known (see, e.g., [61]) and provide the necessary data for a description of the neutrino scattering by the stepping formula (55) down to the values of the energy transfer $T$ in the range of the binding of the $M$ electrons, that is, at $T>\left|E_{M}\right| \approx 0.18 \mathrm{keV}$. The corresponding steps in the atomic factor are shown in Figure 5. One can see that the stepping atomic factor (55) mimics upon average over the energy intervals between the electron shells in germanium the Thomas-Fermi result. Thus, it can be considered as refinement of the latter due to accounting for the quantization of the electron binding energies. It can be mentioned that if one applies formulas of Section 4.1 to the onset of the $K$ shell step, namely, just above $10.9 \mathrm{keV}$, the difference from the shown in the plot step function would be practically invisible in the scale of Figure 5.

5.3. Ab Initio Approaches. While the treatments based on a generic model of many-electron atomic targets allow determining characteristic features and behaviors of the differential cross sections (31) and (32), to obtain accurate numerical results one needs to resort to $a b$ initio calculations. Such calculations can be realized using the Hartree-Fock (HF) method (see, e.g., [58]) and its modifications. In the HF approximation, atomic electrons occupy one-electron states in a spherically-symmetric mean-field potential which is derived self-consistently from the solution of the HF equations. Accordingly, each one-electron state independently contributes to the atomic-ionization process. For the first time this approach was formulated in [38, 39], where it was illustrated with numerical calculations of neutrino-impact ionization of the $\mathrm{F}$ and Mo atomic targets. The wave functions and energies of atomic bound states were calculated within the relativistic HF method [62, 63] with local exchangecorrelation potential [64]. The wave functions of outgoing electrons were obtained by a numerical solution of the Dirac equation in the same mean-field potential as for the wave functions of discrete states. Performed in [40] numerical calculations for ionization of the iodine atoms by impact of reactor antineutrinos led the authors to suggest the stepping approximation (55).

In a very recent theoretical study [60], the authors adopted the multiconfiguration relativistic random-phase approximation (MCRRPA) $[65,66]$ to evaluate the germanium atomic factors. This particular method is based on the time-dependent HF approximation [67]; however, several important features make it a better tool beyond the usual HF approximation to describe transitions of open-shell atoms of high atomic number $Z$. First, for open-shell atoms, typically there is more than one configuration which has the desired ground-state properties. Therefore, a proper HF reference state should be formed by a linear combination of these allowed configurations, that is, a multiconfiguration reference state. Second, for atoms of high $Z$, the relativistic corrections can no longer be ignored. By using a Dirac equation, instead of a Schrödinger one, the leading relativistic terms in the atomic Hamiltonian are treated nonperturbatively from the onset. Third, two-body correlations in addition to the $\mathrm{HF}$ approximation are generally important for excited states and transition matrix elements. The randomphase approximation (RPA) is devised to account for part of the additional two-body correlations (particles can be in the valence or core states) not only for the excited but also for the reference state, and in a lot of cases, it gives good agreement with experiment [68]. Furthermore, it has been shown that RPA equations preserve gauge invariance [69]; this provides a measure of stability of their solutions.

The MCRRPA has been applied successfully to photoexcitation and photoionization of divalent atoms such as Be, $\mathrm{Mg}, \mathrm{Zn}$, and $\mathrm{Sr}$ (some of the results are summarized in [70]). Following similar treatments, the authors of [60] treated the electronic configuration of germanium as a core filled up to the $4 s$ orbits, with two valence electrons in the $4 p$ orbits. As the Ge ground state is a ${ }^{3} P_{0}$ state, it is a linear combination of two configurations, namely, [Zn] $4 p_{1 / 2}^{2}$ and $[\mathrm{Zn}] 4 p_{3 / 2}^{2}$. The wave function was calculated using the multiconfiguration Dirac-Fock package [71]. The atomic excitations due to 


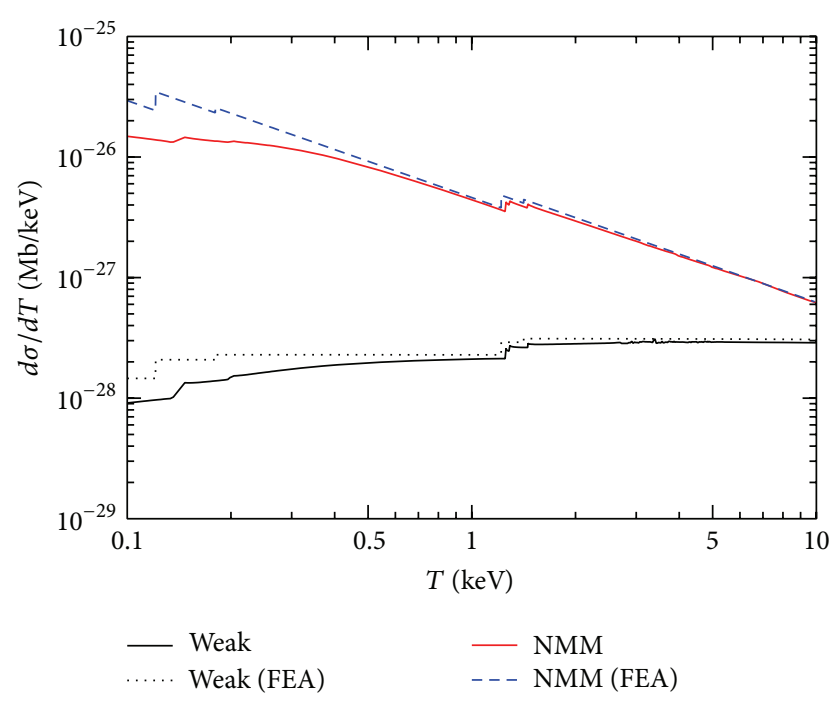

FIGURE 6: The SM (weak) and NMM contributions to the differential cross section of $\bar{\nu}_{e}$-Ge ionization at $E_{v}=1 \mathrm{MeV}$ [60] in comparison with the corresponding stepping-approximation results (FEA). The $\mathrm{NMM}$ value is set to be the current upper limit $\mu_{v}=2.9 \times 10^{-11} \mu_{B}$ [19].

weak and magnetic scattering were solved by the MCRRPA equation, and consequently transition matrix elements were yielded. In that calculation, all the current operators were expanded by spherical multipoles, and the resulting final scattering states were represented in the spherical wave basis and subject to the incoming-wave boundary condition.

Compared with the previous works on the same subject [38-41, 43] which are also in the similar spirit of the relativistic HF method, the MCRRPA approach differs in several respects. First, due to the near degeneracy of the $N_{\text {II }}\left(4 p_{3 / 2}\right)$ and $N_{\text {III }}\left(4 p_{1 / 2}\right)$ levels, using a multiconfiguration reference state is necessary. Second, the nonlocal Fock term is treated exactly, without resorting to the local exchange potentials. Third, the excited states are calculated with twobody correlation built in by MCRRPA, not simply by solving a Coulomb wave function with a static one-hole mean field.

Figure 6 shows numerical results from [60] for ionization of germanium by impact of an electron antineutrino. As can be seen, in the energy region $T \gtrsim 1 \mathrm{keV}$ the results are very well explained by the stepping-approximation formula (55). At the same time, in the sub-keV region, that is, where the electrons from the $K$ and $L$ shells in germanium stay "inactive," both the SM and NMM contributions appear to be significantly suppressed as compared to the stepping approximation. The latter finding seems to disagree with the semiclassical approach discussed in Section 4.2, according to which the ionization involving more loosely-bound electron states, such as those belonging to the $M$ and $N$ shells, is expected to follow more closely the free-electron scenario. Notice that a similar suppression of the atomic-factor values close to the ionization threshold was observed in the case of helium (see Figure 4), and it was attributed to the twoelectron correlation effect. Thus, we can suggest that the correlation effects beyond the approximation of independent electrons lead to the suppression of atomic factors in the lowenergy region. This feature will be important for the nextgeneration experiments with Ge detectors having energy thresholds in the sub-keV region [34-36].

\section{Summary and Perspectives}

In this review, we have considered the neutrino-atom ionizing collisions with focus on the most important theoretical issues related to the problem. The main results discussed in the paper can be summed up as follows.

The differential over the energy transfer cross section given by the free-electron formulas (8) and (9) and the stepping behavior of the atomic factor given by (55) provide a reasonable description of the neutrino-impact ionization of a complex atom, such as germanium, down to quite lowenergy transfer. The deviation from this approximation due to the onset of the ionization near the threshold is less than $5 \%$ (of the height of the step) for the $K$ electrons, if one applies the analytical behavior of this onset that one finds for the ground state of a hydrogen-like ion. It is also found that the free-electron expressions for the differential cross section are not affected by the atomic binding effects in the semiclassical limit and for independent electrons. These analytical results can support the numerically determined behaviors of the electroweak and magnetic contributions to the neutrinoimpact ionization of various atomic targets within the meanfield model [38-41]. At the same time, very recent numerical calculations of the $\bar{v}_{e}$-impact ionization processes of helium [52] and germanium [60] exhibit suppression of the SM and NMM differential cross sections relative to the stepping approximation with lowering the energy-transfer value. This suppression can be assigned to the electron-electron correlation effects.

A theoretical analysis [52] of ionization of helium by electron-antineutrino impact shows no evidence of the recently predicted enhancement [51] of the electromagnetic contribution as compared with the free-electron case. In contrast, in line with previous studies on other atomic targets, it is found that the magnitudes of the differential cross sections decrease relative to the free-electron approximation when the energy transfer is close to the ionization threshold. Thus, no sensitivity enhancement can be expected when using the $\mathrm{He}$ atomic target in searches for NMM. And the stepping approximation appears to be valid, within a few-percent accuracy, down to the energy-transfer values as low as almost $100 \mathrm{eV}$. We thus conclude that for practical applications, that is, for the analysis of data of the searches for NMM, one can safely apply the free-electron formulas and the stepping approximation at the energy transfer down to this range.

When analyzing the low- $T$ data of the current high-sensitivity experiments searching for neutrino electromagnetic properties, one must go beyond the free-electron approximation for the elastic neutrino-electron scattering and take into account the atomic-ionization effects, at least, in the case of $K$ electrons. At the present time, the experiment GEMMA-II with reactor antineutrinos is in preparation [19]. Its sensitivity to the NMM value is expected to be at the level 
of $1 \times 10^{-11} \mu_{B}$. To achieve such a sensitivity level, which is the region of astrophysical interest [24], it is planned to reduce the effective energy threshold of a Ge detector from 2.8 to $1.5 \mathrm{keV}$. This threshold value will be very close to the binding energies of the $L$ electrons in germanium (1.2-1.4 keV [61]). Recently, a $p$-type point-contact Ge detector [34-36] has been implemented in the TEXONO experiment with reactor antineutrinos. The energy threshold of this detector is about $0.3 \mathrm{keV}$, whose value is comparable to the binding energies of the $M_{\text {I-III }}$ electrons in germanium $(0.12-0.18 \mathrm{keV}$ [61]). This means that an accurate analysis of the corresponding data will require numerical calculations based on the ab initio methods.

With lowering the $T$ value down to $T=2 E_{\gamma}^{2} /\left(E_{v}+M_{N}\right)$, an additional collision channel apart from ionization opens up, namely, the coherent elastic neutrino-nucleus scattering [72], which has not been observed experimentally so far. The early treatments of the atomic effects in the coherent elastic neutrino-nucleus scattering within SM can be found in $[37,73,74]$. It should be noted that any deviation of the measured cross section of the coherent elastic neutrinonucleus scattering from the very precisely known SM value [75] will provide a signature of the BSM physics (see [7679]). In this context, the accurate calculations of the NMMinduced contribution to the cross section of the coherent elastic neutrino-nucleus scattering appear to be of particular importance. The NMM-induced coherent neutrino scattering by single atoms as well as by crystals was discussed theoretically only in [80]. However, further studies are necessary for the correct interpretation of future measurements at low $T$ values [81].

\section{Conflict of Interests}

The authors declare that there is no conflict of interests regarding the publication of this paper.

\section{Acknowledgment}

This work was partially supported by the Russian Foundation for Basic Research (Grants no. 14-01-00420-a and no. 14-2203043-ofi-m).

\section{References}

[1] C. Giunti and C. W. Kim, Fundamentals of Neutrino Physics and Astrophysics, Oxford University Press, Oxford, UK, 2007.

[2] M. C. Gonzalez-Garcia and M. Maltoni, "Phenomenology with massive neutrinos," Physics Reports, vol. 460, no. 1-3, pp. 1-129, 2008.

[3] S. Bilenky, Introduction to the Physics of Massive and Mixed Neutrinos, vol. 817 of Lecture Notes in Physics, Springer, New York, NY, USA, 2010.

[4] Z. Z. Xing and S. Zhou, Neutrinos in Particle Physics, Astronomy and Cosmology, Zhejiang University Press, 2011.

[5] C. Giunti and A. Studenikin, "Neutrino electromagnetic properties," Physics of Atomic Nuclei, vol. 72, no. 12, pp. 2089-2125, 2009.
[6] C. Broggini, C. Giunti, and A. Studenikin, "Electromagnetic properties of neutrinos," Advances in High Energy Physics, vol. 2012, Article ID 459526, 47 pages, 2012.

[7] C. Giunti and A. Studenikin, "Neutrino electromagnetic interactions: a window to new physics," http://arxiv.org/abs/1403 .6344 .

[8] J. F. Nieves, "Electromagnetic properties of Majorana neutrinos," Physical Review D, vol. 26, no. 11, pp. 3152-3158, 1982.

[9] B. Kayser, "Majorana neutrinos and their electromagnetic properties," Physical Review D, vol. 26, no. 7, pp. 1662-1670, 1982.

[10] B. Kayser, "CPT, CP, and C phases, and their effects, in Majorana-particle processes," Physical Review D, vol. 30, no. 5, pp. 1023-1033, 1984.

[11] S. T. Petcov, "The processes $\mu \rightarrow e+\gamma, \mu \rightarrow e+e+\bar{e}, \nu^{\prime} \rightarrow \nu+$ $\gamma$ in the Weinberg-Salam model with neutrino mixing," Soviet Journal of Nuclear Physics, vol. 25, p. 340, 1977.

[12] K. Fujikawa and R. E. Shrock, "Magnetic moment of a massive neutrino and neutrino-spin rotation," Physical Review Letters, vol. 45, no. 12, pp. 963-966, 1980.

[13] P. B. Pal and L. Wolfenstein, "Radiative decays of massive neutrinos," Physical Review D, vol. 25, no. 3, pp. 766-773, 1982.

[14] R. E. Shrock, "Electromagnetic properties and decays of Dirac and Majorana neutrinos in a general class of gauge theories," Nuclear Physics B, vol. 206, no. 3, pp. 359-379, 1982.

[15] J. F. Beacom and P. Vogel, "Neutrino magnetic moments, flavor mixing, and the super-kamiokande solar data," Physical Review Letters, vol. 83, no. 25, pp. 5222-5225, 1999.

[16] N. F. Bell, V. Cirigliano, M. J. Ramsey-Musolf, P. Vogel, and M. B. Wise, "How magnetic is the dirac neutrino?" Physical Review Letters, vol. 95, no. 15, Article ID 151802, 4 pages, 2005.

[17] N. F. Bell, M. Gorchtein, M. J. Ramsey-Musolf, P. Vogel, and P. Wang, "Model independent bounds on magnetic moments of Majorana neutrinos," Physics Letters B: Nuclear, Elementary Particle and High-Energy Physics, vol. 642, no. 4, pp. 377-383, 2006.

[18] H. T. Wong, H. B. Li, S. T. Lin et al., "Search of neutrino magnetic moments with a high-purity germanium detector at the KuoSheng nuclear power station," Physical Review D, vol. 75, no. 1 , Article ID 012001, 16 pages, 2007.

[19] A. G. Beda, V. B. Brudanin, V. G. Egorov et al., "The results of search for the neutrino magnetic moment in GEMMA experiment," Advances in High Energy Physics, vol. 2012, Article ID 350150, 12 pages, 2012.

[20] L. B. Auerbach, R. L. Burman, D. O. Caldwell et al., "Measurement of electron-neutrino electron elastic scattering," Physical Review D, vol. 63, no. 11, Article ID 112001, 2001.

[21] R. Schwienhorst, D. Ciampa, C. Erickson et al., "A new upper limit for the tau-neutrino magnetic moment," Physics Letters B, vol. 513, no. 1-2, pp. 23-29, 2001.

[22] D. W. Liu, Y. Ashie, S. Fukuda et al., "Limits on the neutrino magnetic moment using 1496 days of Super-Kamiokande-I solar neutrino data," Physical Review Letters, vol. 93, no. 2, Article ID 021802, 5 pages, 2004.

[23] C. Arpesella, H. O. Back, and M. Balata, "Direct measurement of the 7Be solar neutrino flux with 192 days of Borexino data," Physical Review Letters, vol. 101, no. 9, Article ID 091302, 6 pages, 2008.

[24] G. G. Raffelt, "New bound on neutrino dipole moments from globular-cluster stars," Physical Review Letters, vol. 64, no. 24, pp. 2856-2858, 1990. 
[25] R. N. Mohapatra and P. B. Pal, Massive Neutrinos in Physics and Astrophysics, vol. 72 of Lecture Notes in Physics, World Scientific, River Edge, NJ, USA, 3rd edition, 2004.

[26] E. D. Commins and P. H. Bucksbaum, Weak Interactions of Leptons and Quarks, Cambridge University Press, Cambridge, UK, 3rd edition, 1983.

[27] J. F. Carlson and J. R. Oppenheimer, "The impacts of fast electrons and magnetic neutrons," Physical Review, vol. 41, no. 6, pp. 763-792, 1932.

[28] H. Bethe, "Ionization power of a neutrino with magnetic moment," Mathematical Proceedings of the Cambridge Philosophical Society, vol. 31, no. 1, pp. 108-115, 1935.

[29] A. V. Kyuldjiev, "Searching for effects of neutrino magnetic moments at reactors and accelerators," Nuclear Physics, Section B, vol. 243, no. 3, pp. 387-397, 1984.

[30] G. Domogatsky and D. Nadezhin, "Modern theory of star evolution and experiments of F. Reines on anti $v e$-scattering detection," Soviet Journal of Nuclear Physics, vol. 12, p. 678, 1971.

[31] C. L. Cowan and F. Reines, "Neutrino magnetic moment upper limit," Physical Review, vol. 107, no. 2, pp. 528-530, 1957.

[32] C. L. Cowan Jr., F. Reines, and F. B. Harrison, "Upper limit on the neutrino magnetic moment," Physical Review, vol. 96, no. 5, article 1294, 1954.

[33] P. Vogel and J. Engel, "Neutrino electromagnetic form factors," Physical Review D, vol. 39, no. 11, pp. 3378-3383, 1989.

[34] H. T. Wong, "Low energy neutrino and dark matter physics with sub-kev germanium detectors," International Journal of Modern Physics D, vol. 20, no. 8, pp. 1463-1470, 2011.

[35] H. B. Li, H. Y. Liao, S. T. Lin et al., "Limits on spin-independent couplings of wimp dark matter with a p-type point-contact germanium detector," Physical Review Letters, vol. 110, no. 26, Article ID 261301, 5 pages, 2013.

[36] H. B. Li, L. Singh, M. K. Singh et al., "Differentiation of bulk and surface events in $p$-type point-contact germanium detectors for light WIMP searches," Astroparticle Physics, vol. 56, pp. 1-8, 2014.

[37] Y. V. Gaponov, Y. L. Dobrynin, and V. N. Tikhonov, "Neutrino and antineutrino scattering on hydrogen-like atom," Soviet Journal of Nuclear Physics, vol. 22, no. 2, pp. 328-334, 1975.

[38] V. Y. Dobretsov, A. B. Dobrotsvetov, and S. A. Fayans, "Inelastic neutrino scattering by atomic electrons," Soviet Journal of Nuclear Physics, vol. 55, p. 1180, 1992.

[39] S. A. Fayans, V. Y. Dobretsov, and A. B. Dobrotsvetov, "Effect of atomic binding on inelastic ve scattering," Physics Letters, Section B: Nuclear, Elementary Particle and High-Energy Physics, vol. 291, no. 1-2, pp. 1-6, 1992.

[40] V. I. Kopeikin, L. A. Mikaelyan, V. V. Sinev, and S. A. Fayans, "Scattering of reactor antineutrinos by electrons," Physics of Atomic Nuclei, vol. 60, no. 11, pp. 1859-1864, 1997.

[41] S. A. Fayans, L. A. Mikaelyan, and V. V. Sinev, "Weak and magnetic inelastic scattering of antineutrinos on atomic electrons," Physics of Atomic Nuclei, vol. 64, no. 8, pp. 1475-1480, 2001.

[42] G. J. Gounaris, E. A. Paschos, and P. I. Porfyriadis, "The ionization of $\mathrm{H}, \mathrm{He}$ or $\mathrm{Ne}$ atoms using neutrinos or antineutrinos at keV energies," Physics Letters B, vol. 525, no. 1-2, pp. 63-70, 2002.

[43] V. I. Kopeikin, L. A. Mikaelyan, and V. V. Sinev, "Inelastic scattering of tritium-source antineutrinos on electrons of germanium atoms," Physics of Atomic Nuclei, vol. 66, no. 4, pp. 707711, 2003.
[44] H. T. Wong, H. Li, and S. Lin, "Enhanced sensitivities for the searches of neutrino magnetic moments through atomic ionization," Physical Review Letters, vol. 105, no. 6, Article ID 061801, 2010.

[45] M. Voloshin, "Neutrino scattering on atomic electrons in searches for the neutrino magnetic moment," Physical Review Letters, vol. 105, no. 20, Article ID 201801, 4 pages, 2010.

[46] H. T. Wong, H.-B. Li, and S.-T. Lin, "Enhanced sensitivities for the searches of neutrino magnetic moments through atomic ionization," Physical Review Letters, vol. 105, Article ID 061801, 2074.

[47] K. A. Kouzakov and A. I. Studenikin, "Magnetic neutrino scattering on atomic electrons revisited," Physics Letters B: Nuclear, Elementary Particle and High-Energy Physics, vol. 696, no. 3, pp. 252-256, 2011.

[48] K. A. Kouzakov and A. I. Studenikin, "Electromagnetic neutrino-atom collisions: the role of electron binding," Nuclear Physics B: Proceedings Supplements, vol. 217, no. 1, pp. 353-356, 2011.

[49] K. A. Kouzakov, A. I. Studenikin, and M. B. Voloshin, “Testing neutrino magnetic moment in ionization of atoms by neutrino impact," JETP Letters, vol. 93, no. 11, pp. 623-627, 2011.

[50] K. A. Kouzakov, A. I. Studenikin, and M. B. Voloshin, "Neutrino-impact ionization of atoms in searches for neutrino magnetic moment," Physical Review D, vol. 83, no. 11, Article ID 113001, 11 pages, 2011.

[51] V. P. Martemyanov and V. G. Tsinoev, "Ionization of helium atoms under the effect of the antineutrino magnetic moment," Physics of Atomic Nuclei, vol. 74, no. 12, pp. 1671-1675, 2011.

[52] K. A. Kouzakov and A. I. Studenikin, "On sensitivity of neutrino-helium ionizing collisions to neutrino magnetic moments," Physics of Particles and Nuclei Letters, vol. 11, no. 4, pp. 458-461, 2014.

[53] L. van Hove, "Correlations in space and time and born approximation scattering in systems of interacting particles," Physical Review, vol. 95, pp. 249-262, 1954.

[54] U. Fano, "Penetration of protons, alpha particles, and mesons," Annual Review of Nucler Science, vol. 13, pp. 1-66, 1963.

[55] D. Belkic, "Bound-free non-relativistic transition form factors in atomic hydrogen," Journal of Physics B: Atomic and Molecular Physics, vol. 14, no. 12, article 005, pp. 1907-1914, 1981.

[56] A. R. Holt, "Matrix elements for bound-free transitions in atomic hydrogen," Journal of Physics B: Atomic and Molecular Physics, vol. 2, no. 11, article 311, pp. 1209-1213, 1969.

[57] A. I. Akhiezer and V. B. Berestetskii, Quantum Electrodynamics, Wiley, New York, 2nd edition, 1965.

[58] L. D. Landau and E. M. Lifshits, Quantum Mechanics: NonRelativistic Theory, Pergamon, Oxford, UK, 3rd edition, 1977.

[59] K. A. Kouzakov, S. A. Zaytsev, Y. V. Popov, and M. Takahashi, "Singly ionizing 100-MeV/amu $C^{6}+\mathrm{He}$ collisions with small momentum transfer," Physical Review A: Atomic, Molecular, and Optical Physics, vol. 86, no. 3, Article ID 032710, 8 pages, 2012.

[60] J.-W. Chen, H.-C. Chi, K.-N. Huang et al., "Atomic ionization of germanium by neutrinos from an ab initio approach," Physics Letters B, vol. 731, pp. 159-162, 2014.

[61] B. L. Henke, E. M. Gullikson, and J. C. Davis, "X-ray interactions: photoabsorption, scattering, transmission, and reflection at $E=50-30,000 \mathrm{eV}, Z=1-92$," Atomic Data and Nuclear Data Tables, vol. 54, no. 2, pp. 181-342, 1993.

[62] I. P. Grant, "Relativistic self-consistent fields," Proceedings of the Royal Society A, vol. 262, pp. 555-576, 1961. 
[63] I. P. Grant, "Relativistic self-consistent fields," Proceedings of the Physical Society, vol. 86, pp. 523-527, 1965.

[64] V. L. Moruzzi, J. F. Janak, and A. R. Williams, Calculated Electronic Properties of Metals, Pergamon, Oxford, UK, 1978.

[65] K.-N. Huang and W. R. Johnson, "Multiconfiguration relativistic random-phase approximation: theory," Physical Review A, vol. 25, no. 2, pp. 634-649, 1982.

[66] K.-N. Huang, "Relativistic many-body theory of atomic transitions. The relativistic equation-of-motion approach," Physical Review A, vol. 26, no. 2, pp. 734-739, 1982.

[67] P. Jorgensen, "Molecular and atomic applications of timedependent Hartree-Fock theory," Annual Review of Physical Chemistry, vol. 26, pp. 359-380, 1975.

[68] M. Ya. Amusia and N. A. Cherepkov, "Many-body electron correlations in scattering processes," Case Studies in Atomic Physics, vol. 5, pp. 47-179, 1975.

[69] D. L. Lin, "Gauge properties of the Hartree-Fock and randomphase approximations," Physical Review A, vol. 16, no. 2, pp. 600-605, 1977.

[70] K.-N. Huang, H.-C. Chi, and H.-S. Chou, "The MCRRPA theory and its applications to photoexcitation and photoionization," Chinese Journal of Physics, vol. 33, no. 6, pp. 565-644, 1995.

[71] J. P. Desclaux, "A multiconfiguration relativistic DIRAC-FOCK program," Computer Physics Communications, vol. 9, no. 1, pp. 31-45, 1975.

[72] D. Z. Freedman, "Coherent effects of a weak neutral current," Physical Review D, vol. 9, no. 5, pp. 1389-1392, 1974.

[73] Yu. V. Gaponov and V. N. Tikhonov, "Elastic scattering of lowenergy neutrinos by atomic systems," Soviet Journal of Nuclear Physics, vol. 26, p. 314, 1977.

[74] L. M. Sehgal and M. Wanninger, "Atomic effects in coherent neutrino scattering," Physics Letters B, vol. 171, no. 1, pp. 107-112, 1986.

[75] A. Drukier and L. Stodolsky, "Principles and applications of a neutral-current detector for neutrino physics and astronomy," Physical Review D, vol. 30, no. 11, pp. 2295-2309, 1984.

[76] K. Scholberg, "Prospects for measuring coherent neutrinonucleus elastic scattering at a stopped-pion neutrino source," Physical Review D, vol. 73, no. 3, Article ID 033005, 9 pages, 2006.

[77] J. Barranco, O. G. Miranda, and T. I. Rashba, "Probing new physics with coherent neutrino scattering off nuclei," Journal of High Energy Physics, vol. 2005, article 021, 14 pages, 2005.

[78] J. Barranco, O. G. Miranda, and T. I. Rashba, "Sensitivity of low energy neutrino experiments to physics beyond the standard model," Physical Review D, vol. 76, Article ID 073008, 9 pages, 2007.

[79] S. Davidson, C. Peña-Garay, N. Rius, and A. Santamaria, "Present and future bounds on non-standard neutrino interactions," Journal of High Energy Physics, vol. 3, no. 3, article 011, 2003.

[80] J. Augustin, B. Müller, and W. Greiner, "Magnetic neutrino scattering by crystals," Physical Review D, vol. 41, no. 5, pp. 16831686, 1990.

[81] J. A. Formaggio, E. Figueroa-Feliciano, and A. J. Anderson, "Sterile neutrinos, coherent scattering, and oscillometry measurements with low-temperature bolometers," Physical Review D, vol. 85, no. 1, Article ID 013009, 2012. 

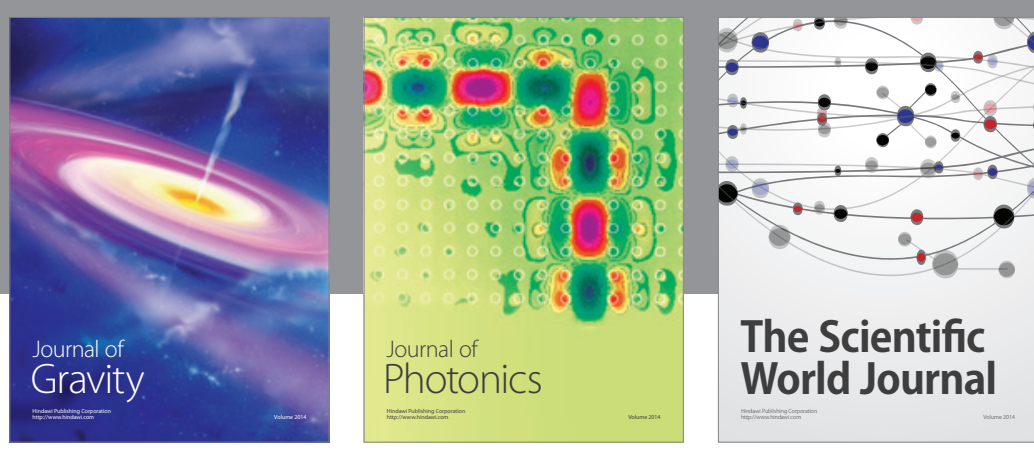

The Scientific World Journal
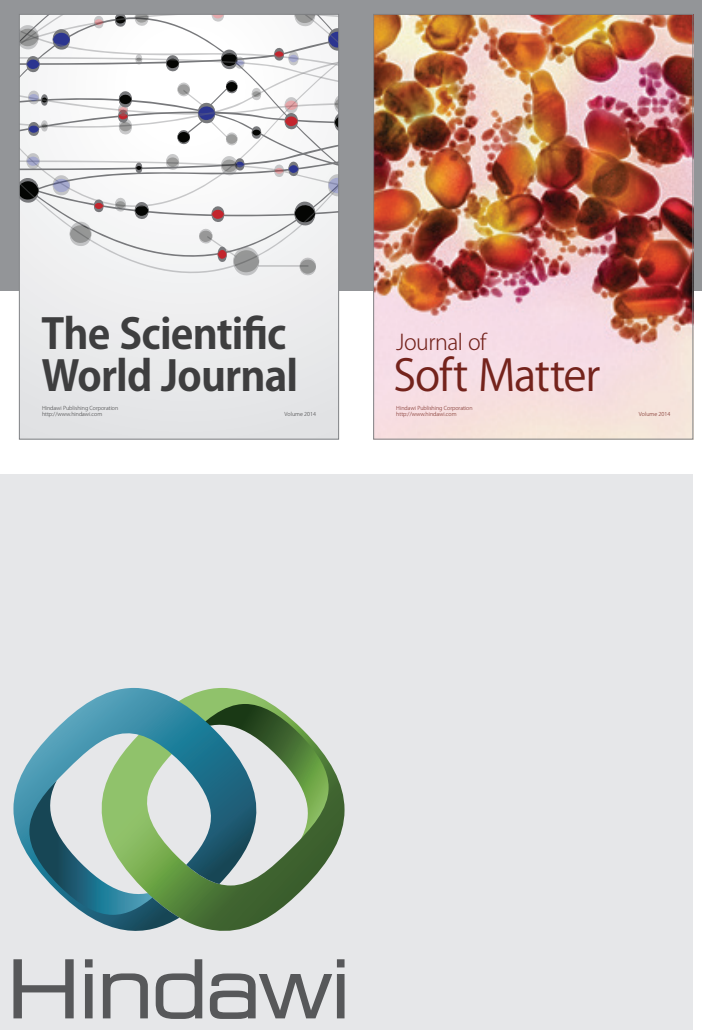

Submit your manuscripts at

http://www.hindawi.com

nternational Journal of

Statistical Mechanics
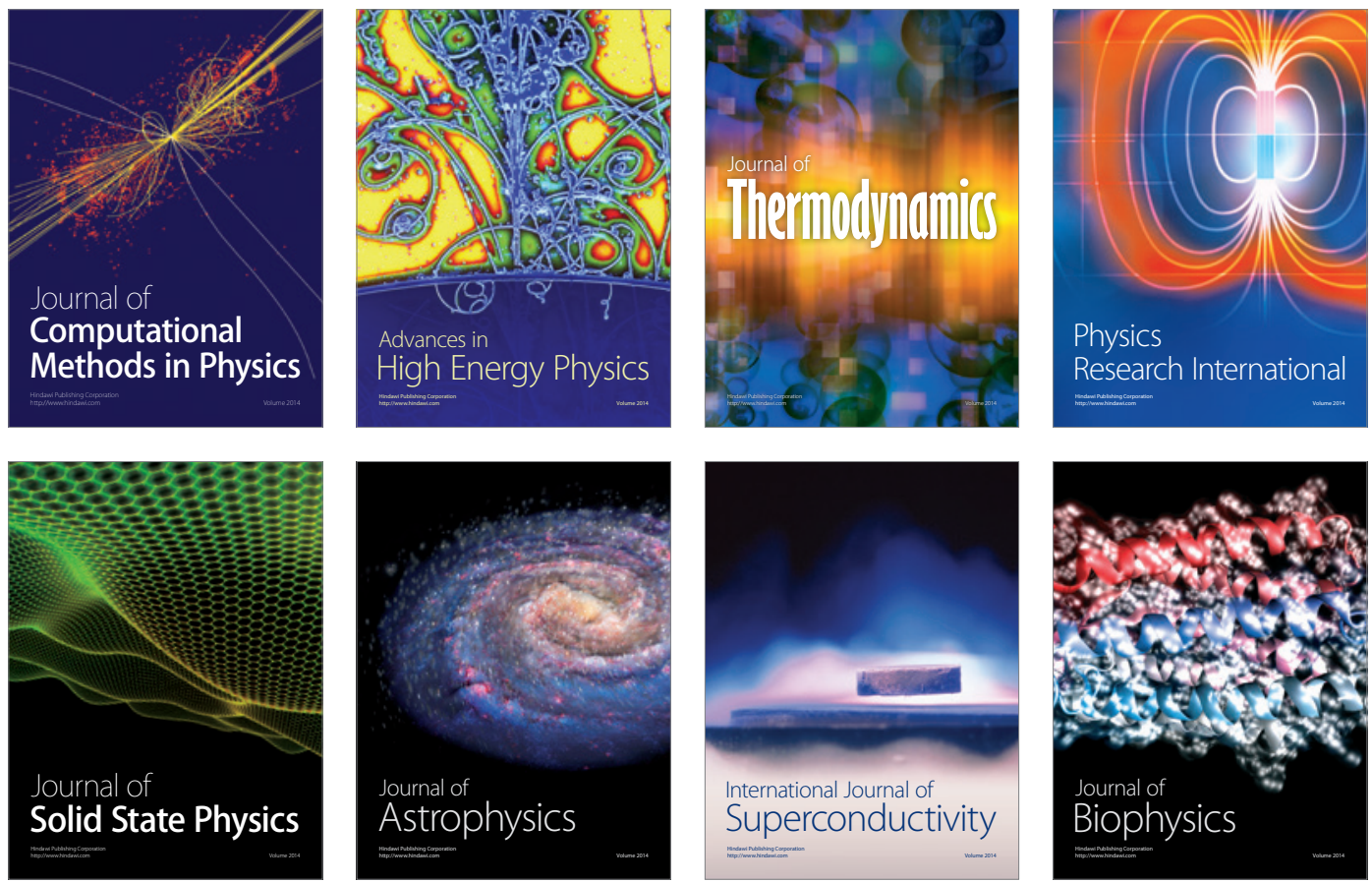
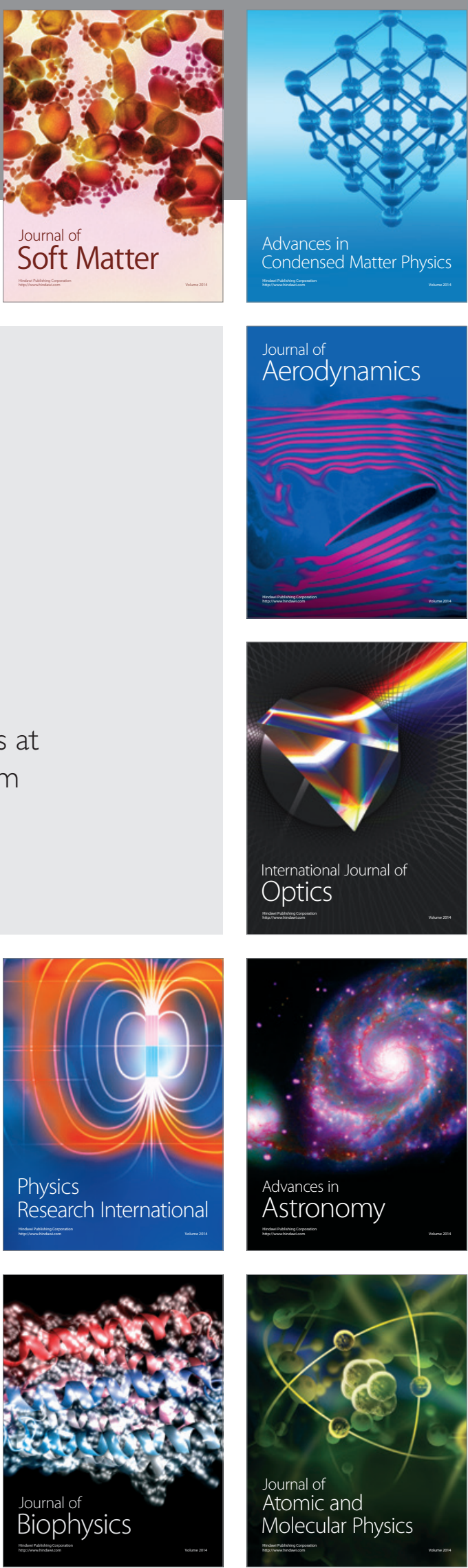